\title{
NONSMOOTH OPTIMAL REGULATION AND DISCONTINUOUS STABILIZATION
}

\author{
A. BACCIOTTI AND F. CERAGIOLI
}

Received 28 October 2002

For affine control systems, we study the relationship between an optimal regulation problem on the infinite horizon and stabilizability. We are interested in the case the value function of the optimal regulation problem is not smooth and feedback laws involved in stabilizability may be discontinuous.

\section{Introduction}

We are interested in the relationship between an optimal regulation problem on the infinite horizon and the stabilization problem for systems affine in the control. This relationship is very well understood in the case of the quadratic regulator for linear systems, where the value function turns out to be quadratic (see, e.g., [2, 18, 28], and [10] for infinite-dimensional systems). The generalization of the linear framework to nonlinear affine systems has been studied in the case the value function of the optimal regulation problem is at least $C^{1}$ (see $[8,25,26,29,33])$. The main purpose of this paper is to relax this regularity assumption; more precisely, we assume that the value function is locally Lipschitz continuous. In particular, we investigate to what extent and in what sense solvability of the optimal regulation problem still implies stabilizability. We mention that a very preliminary study of this subject was already performed in [6].

Essential tools for our extension are nonsmooth analysis (especially, the notion of viscosity solution and Clarke gradient) and the theory of differential equations with discontinuous right-hand side. We recall that viscosity solutions have been used in $[23,24]$ in order to obtain stabilizability via optimal regulation. However, in $[23,24]$, the author limits himself to homogeneous systems.

Some results of the present paper hold under additional conditions: somewhere we will assume that the value function is $C$-regular, somewhere else we will make the weaker assumption that it is nonpathological (these properties are defined in Appendix A). Although sufficient conditions for $C$-regularity are not 
known, we present some reasonable examples where the candidate value function is $C$-regular (but not differentiable). We also point out that if the dynamics are linear and the cost is convex, then the value function is convex (and hence C-regular).

Some of our examples involve semiconcave value functions. Semiconcavity appears frequently in optimization theory $[11,17]$. In fact, semiconcavity and $C$-regularity are somehow alternative and can be interpreted as dual properties. As a common feature, both $C$-regular and semiconcave functions turn out to be nonpathological.

In a nonsmooth context, stabilization is often performed by means of discontinuous feedback. To this respect, we remark that in this paper solutions of differential equations with a discontinuous right-hand side are intended either in Carathéodory sense or in Filippov senses. In some recent papers [14, 15, 31], interesting work has been done by using different approaches (proximal analysis and sampling).

When the value function is of class $C^{1}$, stabilization via optimal regulation guarantees robustness and stability margin for the control law (to this respect, see $[22,37]$ and especially [33]). The robustness issue is not addressed in the present paper; however, our results indicate that such a development may be possible even in the nonsmooth case.

We now describe more precisely the two problems we deal with.

1.1. Feedback stabilization. We consider a system of the form

$$
\dot{x}=f(x)+G(x) u=f(x)+\sum_{i=1}^{m} u_{i} g_{i}(x),
$$

where $x \in \mathbb{R}^{n}, u \in \mathbb{R}^{m}$, the vector fields $f: \mathbb{R}^{n} \rightarrow \mathbb{R}^{n}, g_{i}: \mathbb{R}^{n} \rightarrow \mathbb{R}^{n}, i=1, \ldots, m$, are of class $C^{1}$, and $G$ is the matrix whose columns are $g_{1}, \ldots, g_{m}$. For most of the paper, as admissible inputs, we consider piecewise continuous and right continuous functions $u: \mathbb{R} \rightarrow \mathbb{R}^{m}$. We denote by $\boldsymbol{u}$ the set of admissible inputs and by $\varphi(t ; x, u(\cdot))$ the solution of $(1.1)$ corresponding to a fixed control law $u(\cdot) \in \mathcal{U}$ such that $\varphi(0 ; x, u(\cdot))=x$. We remark that for every admissible input and every initial condition there exists a Carathéodory solution which is unique. We require that all such solutions be right continuable on $[0,+\infty)$.

We say that system (1.1) is (globally) stabilizable if there exists a map $u=$ $k(x): \mathbb{R}^{n} \rightarrow \mathbb{R}^{m}$, called a feedback law, such that, for the closed loop system

$$
\dot{x}=f(x)+G(x) k(x)
$$

the following properties hold:

(i) (Lyapunov stability) for all $\epsilon>0$, there exists $\delta>0$ such that for each solution $\varphi(\cdot)$ of (1.2), $|\varphi(0)|<\delta$ implies $|\varphi(t)|<\epsilon$ for all $t \geq 0$,

(ii) (attractivity) for each solution $\varphi(t)$ of (1.2), one has $\lim _{t \rightarrow+\infty} \varphi(t)=0$. 
It is well known that the class of continuous feedbacks is not sufficiently large in order to solve general stabilization problems (see $[3,9,36])$. For this reason, in the following we also consider discontinuous feedbacks. Of course, the introduction of discontinuous feedback laws leads to the theoretical problem of defining solutions of the differential equation (1.2) whose right-hand side is discontinuous. In the following we consider Carathéodory and Filippov solutions (the definition of Filippov solution is recalled in Appendix A; see also [20]). Thus we say that system (1.1) is either Carathéodory or Filippov stabilizable according to the fact that we consider either Carathéodory or Filippov solutions of the closed loop system (1.2).

1.2. The optimal regulation problem. We associate to system (1.1) the cost functional

$$
J(x, u(\cdot))=\frac{1}{2} \int_{0}^{+\infty}\left(h(\varphi(t ; x, u(\cdot)))+\frac{|u(t)|^{2}}{\gamma}\right) d t,
$$

where $h: \mathbb{R}^{n} \rightarrow \mathbb{R}$ is a continuous, radially unbounded function with $h(x) \geq 0$ for all $x$ and $y \in \mathbb{R}^{+}$. Radially unboundedness means that $\lim _{|x| \rightarrow \infty} h(x)=+\infty$; such a property is needed in order to achieve global results, and can be neglected if one is only interested in a local treatment. Occasionally, we will also require that $h$ be positive definite, that is, $h(0)=0$ and $h(x)>0$ if $x \neq 0$.

We are interested in the problem of minimizing the functional $J$ for every initial condition $x$. The value function $V: \mathbb{R}^{n} \rightarrow \mathbb{R}$ associated to the minimization problem is

$$
V(x)=\inf _{u \in \mathscr{U}} J(x, u(\cdot)) .
$$

We say that the optimal regulation problem is solvable if for every $x$ the infimum in the definition of $V$ is actually a minimum. If this is the case, we denote by $u_{x}^{*}(\cdot)$ an optimal open-loop control corresponding to the initial condition $x$; we also write $\varphi_{x}^{*}(\cdot)$ instead of $\varphi\left(t ; x, u_{x}^{*}(\cdot)\right)$.

In the classical approach, it is usual to assume that the value function is of class $C^{1}$. Under this assumption, the following statement is well known: a system for which the optimal regulation problem is solvable can be stabilized by means of a feedback in the so-called damping form

$$
u=k_{\alpha}(x)=-\alpha(\nabla V(x) G(x))^{\mathbf{t}}
$$

(the exponent $\mathbf{t}$ denotes transposition) provided that $\alpha$ is a sufficiently large positive real constant. As already mentioned, in this paper, we are interested in the case the value function is merely locally Lipschitz continuous. This case is particularly interesting because it is known that if $h$ is locally Lipschitz continuous and if certain restrictive assumptions about the right-hand side of (1.1) are fulfilled, then the value function is locally Lipschitz continuous (see [19]). 


\section{Nonsmooth optimal regulation and discontinuous stabilization}

1.3. Plan of the paper and description of the results. In Section 2, we generalize the classical necessary conditions which must be fulfilled by optimal controls and by the value function of an optimal regulation problem. We also provide an expression for an optimal control which is reminiscent of the feedback form (1.5).

The results concerning stabilization are presented in Sections 3 and 4. By combining some well-known results about stabilization of asymptotically controllable systems, with the characterizations of optimal controls given in Section 2 , in Section 3 we first prove that solvability of the optimal regulation problem implies Carathéodory stabilizability. Then, by assuming that the value function is $C$-regular, we prove that the solvability of the optimal regulation problem also implies Filippov stabilizability. Unfortunately, by this way we are not able to recover any explicit form of the feedback law. We are so led to directly investigate the stabilizing properties of the feedback (1.5). To this respect, we prove two theorems in Section 4. Both of them apply when the value function is nonpathological (in the sense introduced by Valadier in [38]). The first one makes use of a strong condition, actually implying that (1.5) is continuous. The second theorem is more general, but requires an additional assumption.

In Section 5, we finally prove a nonsmooth version of the optimality principle (see $[8,25,33])$. It turns out to be useful in the analysis of the illustrative examples presented in Section 6. Particularly interesting are Examples 6.4 and 6.5, which enlighten some intriguing features of the problem.

Two appendices conclude the paper. In Appendix A, we collect some tools of nonsmooth analysis used throughout the paper. These include a new characterization of Clarke regular functions and the proof that semiconcave functions are nonpathological. The proofs of all the results of the present paper are based on several lemmas which are stated and proved in Appendix B.

\section{Necessary conditions for optimality}

It is well known that when the value function is of class $C^{1}$, a necessary (as well as sufficient) condition for optimality can be given in terms of a partial differential equation of the Hamilton-Jacobi type. Moreover, optimal controls admit a representation in the feedback form (1.5), with $\alpha=\gamma$ (see, e.g., [35]). The aim of this section is to prove analogous results for the case the value function is locally Lipschitz continuous. The optimal regulation problem (1.3) is naturally associated with the pre-Hamiltonian function

$$
\mathscr{H}(x, p, u)=-p \cdot(f(x)+G(x) u)-\frac{h(x)}{2}-\frac{|u|^{2}}{2 \gamma}
$$

For each $x$ and $p$, the map $u \mapsto \mathscr{H}(x, p, u)$ is strictly concave. By completing the square, we easily obtain the following expression for the Hamiltonian 
function:

$$
\begin{aligned}
H(x, p) & \stackrel{\text { def }}{=} \max _{u} \mathscr{H}(x, p, u)=\mathscr{H}\left(x, p,-\gamma(p G(x))^{\mathbf{t}}\right) \\
& =-p f(x)+\frac{\gamma}{2}|p G(x)|^{2}-\frac{h(x)}{2} .
\end{aligned}
$$

The achievements of this section are presented in Propositions 2.1 and 2.3. Comments and remarks are inserted in order to relate our conclusions to the existing literature. The proofs are essentially based on the dynamic programming principle (see $[7,35]$ ) and some lemmas established in Appendix B; we also exploit certain tools of nonsmooth analysis (see Appendix A for notations and definitions).

Proposition 2.1. Assume that the optimal regulation problem is solvable and that the value function $V(x)$ is locally Lipschitz continuous. Let $x \in \mathbb{R}^{n}$ be fixed. Let $u_{x}^{*}(\cdot)$ be an optimal control for $x$ and let $\varphi_{x}^{*}(\cdot)$ be the corresponding optimal solution. Then for all $t \geq 0$ there exists $p_{0}(t) \in \partial_{C} V\left(\varphi_{x}^{*}(t)\right)$ such that

(i) $H\left(\varphi_{x}^{*}(t), p_{0}(t)\right)=0$,

(ii) $u_{x}^{*}(t)=-\gamma\left(p_{0}(t) G\left(\varphi_{x}^{*}(t)\right)\right)^{\mathbf{t}}$.

Proof. Lemmas B.1 and B.2 imply that

$$
\forall x \in \mathbb{R}^{n}, \forall t \geq 0, \quad \exists u_{0}(t) \in \mathbb{R}^{m}, \quad \exists p_{0}(t) \in \partial_{C} V\left(\varphi_{x}^{*}(t)\right)
$$

such that $\mathscr{H}\left(\varphi_{x}^{*}(t), p_{0}(t), u_{0}(t)\right)=0$.

On the other hand, by Lemma B.3, $\mathscr{H}\left(\varphi_{x}^{*}(t), p_{0}(t), u\right) \leq 0$ for each $u \in \mathbb{R}^{m}$. Recalling the definition of $H$, (i) and (ii) are immediately obtained.

Remark 2.2. Under the assumptions of Proposition 2.1, we also have

$$
\forall x \in \mathbb{R}^{n}, \quad \exists p_{0} \in \partial_{C} V(x) \quad \text { such that } H\left(x, p_{0}\right)=0 .
$$

This follows from statement (i), setting $t=0$.

Proposition 2.1 is a necessary condition for an open-loop control being optimal. In particular, (ii) provides the analogue of the usual feedback form representation of optimal controls. The following proposition gives necessary conditions for $V(x)$ being the value function of the optimal regulation problem.

Proposition 2.3. Given the optimal regulation problem (1.3), assume that the value function $V(x)$ is locally Lipschitz continuous. Then,

(i) for each $x \in \mathbb{R}^{n}$ and for each $p \in \partial_{C} V(x), H(x, p) \leq 0$.

In addition, assume that the optimal regulation problem is solvable. Then,

(ii) for each $x \in \mathbb{R}^{n}$ and for each $p \in \underline{\partial} V(x), H(x, p)=0$. 
1164 Nonsmooth optimal regulation and discontinuous stabilization

Proof. Statement (i) is an immediate consequence of Lemma B.3 and the definition of $H$; statement (ii) follows by Lemma B.4, taking into account statement (i).

Propositions 2.1 and 2.3 can be interpreted in terms of generalized solutions of the Hamilton-Jacobi equation

$$
H(x, \nabla V(x))=0
$$

Indeed, Proposition 2.3 implies in particular that $V(x)$ is a viscosity solution of (2.5) (a similar conclusion is obtained in [19] for a more general cost functional but under restrictive assumptions on the vector fields). Note that Proposition 2.3(ii) cannot be deduced from [7, Theorem 5.6] since in our case the Hamiltonian function is not uniformly continuous on $\mathbb{R}^{n}$. Together with Proposition 2.3(i), (2.4) can be interpreted by saying that $V(x)$ is a solution in extended sense of $(2.5)$ ( since $p \mapsto H(x, p)$ is convex, the same conclusion also follows from [7, Proposition 5.13]; in fact, we provide a simpler and more direct proof).

Finally, Proposition 2.3(i) implies that $V(x)$ is a viscosity supersolution of the equation

$$
-H(x, \nabla V(x))=0 .
$$

Remark 2.4. In general, it is not true that $V(x)$ is a viscosity subsolution of (2.6), unless certain additional conditions such as $C$-regularity are imposed (see Corollary 2.5). This is the reason why the complete equivalence between solvability of the optimal regulation problem, solvability of the Hamilton-Jacobi equation, and stabilizability by damping feedback breaks down in the general nonsmooth case. Basically, this is the main difference between the smooth and the nonsmooth cases.

If the value function $V(x)$ satisfies additional assumptions, further facts can be proven. For instance, from Propositions 2.3(ii) and A.2, we immediately obtain the following corollary.

Corollary 2.5. Assume that the optimal regulation problem is solvable and let $V(x)$ be the value function. Assume further that $V(x)$ is locally Lipschitz continuous and $C$-regular. Then,

$$
\forall x \in \mathbb{R}^{n}, \forall p \in \partial_{C} V(x), \quad H(x, p)=0 .
$$

Remark 2.6. Corollary 2.5 implies that $V(x)$ is a subsolution of (2.6), as well. Moreover, when $V(x)$ is $C$-regular, in Proposition 2.1(ii), we can choose any $p_{0}(t) \in \partial_{C} V\left(\varphi_{x}^{*}(t)\right)$. 


\section{Control Lyapunov functions and stabilizability}

In this section, we show that the value function of the optimal regulation problem can be interpreted as a control Lyapunov function for system (1.1). Then, by using well-known results in the literature, we will be able to recognize that a system for which the optimal regulation problem is solvable can be stabilized both in Carathéodory and Filippov senses. However, by this approach, it is not possible to give an explicit construction of the feedback law.

Since we consider nonsmooth value functions, our definition of control Lyapunov function must make use of some sort of generalized gradient. Actually, we need two different kinds of control Lyapunov functions, introduced, respectively, by Sontag [36] and Rifford [32]. We denote by $\partial V$ a (for the moment unspecified) generalized gradient of a function $V: \mathbb{R}^{n} \rightarrow \mathbb{R}$.

Definition 3.1. We say that $V: \mathbb{R}^{n} \rightarrow \mathbb{R}^{+}$is a control Lyapunov function for system (1.1) in the sense of the generalized gradient $\partial$ if it is continuous, positive definite, and radially unbounded, and there exist $W: \mathbb{R}^{n} \rightarrow \mathbb{R}$ continuous, positive definite, and radially unbounded, and $\sigma: \mathbb{R}^{+} \rightarrow \mathbb{R}^{+}$nondecreasing such that

$$
\sup _{x \in \mathbb{R}^{n}} \max _{p \in \partial V(x)} \min _{|u| \leq \sigma(|x|)}\{p \cdot(f(x)+G(x) u)+W(x)\} \leq 0,
$$

that is,

$$
\forall x \in \mathbb{R}^{n}, \forall p \in \partial V(x), \quad \exists \bar{u}:|\bar{u}| \leq \sigma(|x|), \quad p \cdot(f(x)+G(x) \bar{u})+W(x) \leq 0 .
$$

In particular, we say that $V(x)$ is a control Lyapunov function in the sense of the proximal subdifferential if $\partial=\partial_{P}$ and we say that $V(x)$ is a control Lyapunov function in the sense of Clarke generalized gradient if $\partial=\partial_{C}$.

3.1. Carathéodory stabilizability. We now prove the Carathéodory stabilizability result. We get it as a consequence of Ancona and Bressan's result (see [1]) which states that an asymptotically controllable system is Carathéodory stabilizable. The expression obtained for the optimal control in Proposition 2.1 also plays an important role. We first recall the definition of asymptotic controllability.

We say that system (1.1) is asymptotically controllable if

(i) for each $x$, there exists an input $u_{x}(\cdot) \in \mathcal{U}$ such that $\lim _{t \rightarrow+\infty} \varphi\left(t ; x, u_{x}(\cdot)\right)$ $=0$,

(ii) for each $\epsilon>0$, there exists $\delta>0$ such that, if $|x|<\delta$, there exists a control $u_{x}(\cdot)$ as in (i) such that $\left|\varphi\left(t ; x, u_{x}(\cdot)\right)\right|<\epsilon$ for each $t \geq 0$.

Moreover, we require that there exist $\delta_{0}>0$ and $\eta_{0}>0$ such that, if $|x|<\delta_{0}$, then $u_{x}(\cdot)$ can be chosen in such a way that $\left|u_{x}(t)\right|<\eta_{0}$ for $t \geq 0$. 
Theorem 3.2. Let system (1.1) be given and let $h(x)$ be continuous, radially unbounded, and positive definite. If the optimal regulation problem (1.3) is solvable and if its value function $V(x)$ is locally Lipschitz continuous and radially unbounded, then $V(x)$ is a control Lyapunov function in the sense of the proximal subdifferential, and the system is asymptotically controllable. Moreover, the system is Carathéodory stabilizable.

Proof. Thanks to [36, Theorem D, page 569], system (1.1) is asymptotically controllable if and only if there exists a control Lyapunov function in the sense of the proximal subdifferential. Thus, the conclusion follows from Lemma B.4 and the fact that $\partial_{P} V(x) \subseteq \underline{\partial} V(x)$.

Note that the existence of $\sigma$ such that $\left|u_{x}^{*}(0)\right| \leq \sigma(|x|)$ is a consequence of the feedback form obtained for the optimal control in Proposition 2.1 and the fact that the set-valued map $\partial_{C} V$ is upper semicontinuous with compact values. The second statement is therefore a consequence of [1, Theorem 1].

We remark that since asymptotic controllability has been proven, stabilizability in the sense of the so-called sampling solutions may also be deduced (see [15]). A different proof of asymptotic controllability which does not make use of [36, Theorem D] was already given in [6]. There, the fact that an optimal control gives asymptotic controllability was proved by means of Lemma B.5. From that proof, it turns out evidently that the optimal control itself gives asymptotic controllability.

3.2. Filippov stabilizability. We now discuss Filippov stabilizability. In this section, we consider the case where the value function $V(x)$ is $C$-regular. The result is based on the interpretation of the value function as a control Lyapunov function in the sense of Clarke generalized gradient. In Section 4 the result will be improved: indeed, we will show that, under the same assumptions, the system can be stabilized just by the damping feedback (1.5) with $\alpha$ large enough.

Theorem 3.3. Let system (1.1) be given and let $h$ be continuous, radially unbounded, and positive definite. If the optimal regulation problem (1.3) is solvable and if its value function $V(x)$ is locally Lipschitz continuous, C-regular, and radially unbounded, then $V(x)$ is a control Lyapunov function in the sense of Clarke gradient. Moreover, the system is Filippov stabilizable.

Proof. The first statement is a trivial consequence of Lemma B.4, the fact that for $C$-regular functions, $\underline{\partial} V(x)=\partial_{C} V(x)$ for all $x$ (see Proposition A.2), and the feedback form obtained for the optimal control in Proposition 2.1. Then, the second statement follows from [32, Theorem 2.7], according to which the existence of a control Lyapunov function in the sense of Clarke gradient guarantees Filippov stabilizability (the differences between our definition of control Lyapunov function in the sense of Clarke generalized gradient and the definition given in [32] are not essential). 
Remark 3.4. Due to [32, Theorem 2.7], the existence of a control Lyapunov function in the sense of Clarke generalized gradient for (1.1) also implies the existence of a $C^{\infty}$ Lyapunov function. In turn, thanks to Sontag universal formula, this implies the existence of a stabilizing feedback in $C^{1}\left(\mathbb{R}^{n} \backslash\{0\}\right)$ (see also [32, Theorem 2.8]).

\section{Stabilization by damping feedback}

As already mentioned, in this section, we improve the result of Theorem 3.3. More precisely, we discuss the possibility of stabilizing the system by means of an explicit feedback in damping form. For a moment, we forget the optimal regulation problem and let $V(x)$ be any locally Lipschitz continuous function. Consider the corresponding feedback law defined by (1.5). When it is implemented, it gives rise to the closed loop system

$$
\dot{x}=f(x)+G(x) k_{\alpha}(x)=f(x)-\alpha G(x)(\nabla V(x) G(x))^{\mathbf{t}} .
$$

In general, the right-hand side of (4.1) is not continuous. Indeed, by virtue of Rademacher's theorem, the right-hand side of (4.1) is almost everywhere defined; moreover, it is locally bounded and measurable (see [5]). Nevertheless, under the assumptions of the next theorem, the feedback law (1.5) turns out to be continuous so that (4.1) possesses solutions in classical sense.

Theorem 4.1. Let $V: \mathbb{R}^{n} \mapsto \mathbb{R}$ be locally Lipschitz continuous, positive definite, and radially unbounded. Let $h: \mathbb{R}^{n} \mapsto \mathbb{R}$ be continuous, positive definite, and radially unbounded. Let $H$ be defined according to (2.2). Assume that

$$
\forall x \in \mathbb{R}^{n}, \forall p \in \partial_{C} V(x), \quad H(x, p)=0
$$

Then, the map $x \mapsto \nabla V(x) G(x)$ admits a continuous extension. If in addition $V(x)$ is positive definite, radially unbounded, and nonpathological, the damping feedback (1.5) with $\alpha \geq \gamma / 2$ is a stabilizer (in classical sense) for system (1.1).

Proof. By contradiction, assume that there exists a point $\bar{x}$, where $\nabla V(x) G(x)$ cannot be completed in a continuous way. There must exist sequences $x_{n}^{\prime} \rightarrow \bar{x}$ and $x_{n}^{\prime \prime} \rightarrow \bar{x}$ such that

$$
\lim _{n} \nabla V\left(x_{n}^{\prime}\right) G\left(x_{n}^{\prime}\right)=c^{\prime} \neq c^{\prime \prime}=\lim _{n} \nabla V\left(x_{n}^{\prime \prime}\right) G\left(x_{n}^{\prime \prime}\right)
$$

Since $V(x)$ is locally Lipschitz continuous, its gradient, where it exists, is locally bounded. Possibly taking subsequences, we may assume that the limits

$$
p^{\prime}=\lim _{n} \nabla V\left(x_{n}^{\prime}\right), \quad p^{\prime \prime}=\lim _{n} \nabla V\left(x_{n}^{\prime \prime}\right)
$$


exist. Of course, $p^{\prime} \neq p^{\prime \prime}$. Clearly, $p^{\prime}, p^{\prime \prime} \in \partial_{C} V(\bar{x})$, and hence, by assumption (4.2),

$$
-p^{\prime} f(\bar{x})+\frac{\gamma}{2}\left|c^{\prime}\right|^{2}-\frac{h(\bar{x})}{2}=0, \quad-p^{\prime \prime} f(\bar{x})+\frac{\gamma}{2}\left|c^{\prime \prime}\right|^{2}-\frac{h(\bar{x})}{2}=0 .
$$

Let $0<\mu, \nu<1$, with $\mu+\nu=1$. From (4.5) it follows that

$$
-p f(\bar{x})+\frac{\gamma}{2}\left[\mu\left|c^{\prime}\right|^{2}+\nu\left|c^{\prime \prime}\right|^{2}\right]-\frac{h(\bar{x})}{2}=0,
$$

where $p=\mu p^{\prime}+v p^{\prime \prime}$. On the other hand, since $\partial_{C} V(\bar{x})$ is convex, invoking again assumption (4.2), we have

$$
\begin{aligned}
0 & =-p f(\bar{x})+\frac{\gamma}{2}|p G(\bar{x})|^{2}-\frac{h(\bar{x})}{2} \\
& <-p f(\bar{x})+\frac{\gamma}{2}\left[\mu\left|c^{\prime}\right|^{2}+\nu\left|c^{\prime \prime}\right|^{2}\right]-\frac{h(\bar{x})}{2}=0,
\end{aligned}
$$

where we also used the fact that the map $c \mapsto|c|^{2}$ is strictly convex. Comparing (4.6) and (4.7), we obtain a contradiction, and the first conclusion is achieved.

The second conclusion is based on the natural interpretation of $V$ as a Lyapunov function for the closed loop system. Although we now know that the right-hand side of such system is continuous, we cannot apply the usual Lyapunov argument since $V$ is not differentiable. Instead, we invoke Proposition A.4 which is stated in terms of the set-valued derivative of a nonpathological function with respect to a differential inclusion.

Let $x$ be arbitrarily fixed $(x \neq 0)$ and let $a \in \dot{\bar{V}}^{(4.1)}(x)$ (the notation is explained in Appendix A). Then $a$ is such that there exists $q \in \partial_{C} V(x)$ such that $a=p \cdot\left(f(x)-(\gamma / 2) G(x)(q G(x))^{\mathbf{t}}\right)$ for all $p \in \partial_{C} V(x)$. We have to prove that $a<0$. If we take $p=q$, we obtain the following expression for $a$ :

$$
a=q \cdot f(x)-\frac{\gamma}{2}|q G(x)|^{2} .
$$

By virtue of assumption (4.2), we get that $a=-h(x) / 2$. Finally, we recall that $h$ is positive definite. The statement is so proved for $\alpha=\gamma / 2$. The case $\alpha>\gamma / 2$ easily follows.

Coming back to the optimal regulation problem and recalling Corollary 2.5, we immediately have the following corollary.

Corollary 4.2. The same conclusion of Theorem 4.1 holds in particular when the optimal regulation problem is solvable and the value function $V(x)$ is locally Lipschitz continuous, C-regular, and radially unbounded.

Remark 4.3. Theorem 3.3 and Corollary 4.2 emphasize the role of $C$-regular functions. To this respect, it would be interesting to know conditions about the 
function $h(x)$, which enable us to prove that $V(x)$ is $C$-regular. The problem seems to be open in general. In Section 6, we show some examples where the function $V(x)$ is $C$-regular. Moreover, we point out some particular (but not completely trivial) situations where convexity (and hence, $C$-regularity and Lipschitz continuity) of $V(x)$ is guaranteed.

Assume for instance that system (1.1) is linear, that is, $f(x)=A x$ and $G(x)=$ $B$, and that $h$ is convex. Let $x_{1}, x_{2} \in \mathbb{R}^{n}$, let $0 \leq \nu$ and $\mu \leq 1$ be such that $\nu+\mu=1$, and let $\varepsilon>0$. We have

$$
\begin{aligned}
\nu V\left(x_{1}\right)+\mu V\left(x_{2}\right)+\varepsilon \geq \frac{1}{2}[ & \int_{0}^{\infty}\left(\nu h\left(\varphi_{x_{1}}^{\varepsilon}(t)\right)+\mu h\left(\varphi_{x_{2}}^{\varepsilon}(t)\right)\right) d t \\
& \left.+\frac{1}{\gamma} \int_{0}^{\infty}\left(\nu\left|u_{x_{1}}^{\varepsilon}(t)\right|^{2}+\mu\left|u_{x_{2}}^{\varepsilon}(t)\right|^{2}\right) d t\right],
\end{aligned}
$$

where, according to the definition of $V, u_{x_{i}}^{\varepsilon}$ is such that $V\left(x_{i}\right)+\varepsilon \geq J\left(x_{i}, u_{x_{i}}^{\varepsilon}\right)$, $i=1,2$. Using the convexity of both $h$ and the quadratic map $u \mapsto|u|^{2}$ yields

$$
\begin{aligned}
\nu V\left(x_{1}\right)+\mu V\left(x_{2}\right)+\varepsilon \geq \frac{1}{2}[ & \int_{0}^{\infty} h\left(\nu \varphi_{x_{1}}^{\varepsilon}(t)+\mu \varphi_{x_{2}}^{\varepsilon}(t)\right) d t \\
& \left.+\frac{1}{\gamma} \int_{0}^{\infty}\left|\nu u_{x_{1}}^{\varepsilon}(t)+\mu u_{x_{2}}^{\varepsilon}(t)\right|^{2} d t\right] .
\end{aligned}
$$

Finally, by virtue of linearity,

$$
\nu V\left(x_{1}\right)+\mu V\left(x_{2}\right)+\varepsilon \geq \frac{1}{2}\left[\int_{0}^{\infty} h\left(\varphi_{\nu x_{1}+\mu x_{2}}(t)\right) d t+\frac{1}{\gamma} \int_{0}^{\infty}|u(t)|^{2} d t\right],
$$

where $u(t)=v u_{x_{1}}^{\varepsilon}(t)+\mu u_{x_{2}}^{\varepsilon}(t)$ and $\varphi_{x}(t)=\varphi(t ; x, u(\cdot))$. Since $V$ is an infimum and the choice of $\varepsilon$ is arbitrary, we conclude

$$
\nu V\left(x_{1}\right)+\mu V\left(x_{2}\right) \geq V\left(\nu x_{1}+\mu x_{2}\right) .
$$

Note that here the existence of solutions of the optimal regulation problem as well as a priori information about the value function are not required.

Theorem 4.4 provides an alternative stabilizability result. Condition (4.2) of Theorem 4.1 is weakened, so that the damping feedback (1.5) is no more expected to be continuous in general. As a consequence, the stability analysis will be carried out in terms of Filippov solutions. Recall that Filippov solutions of (4.1) coincide with the solutions of the differential inclusion

$$
\dot{x} \in f(x)-\alpha G(x)\left(\partial_{C} V(x) G(x)\right)^{\mathbf{t}}
$$

(see $[5,30])$, where the set-valued character of the right-hand side depends on the presence of Clarke gradient. 
Weakening condition (4.2) is balanced by the introduction of a new assumption. Roughly speaking, this new assumption amounts to say that $V$ is not "too irregular" with respect to the vector fields $g_{1}, \ldots, g_{m}$ (in a sense to be precised).

In particular, Theorem 4.4 focuses on the class of nonpathological functions. The definition is given in Appendix A. We recall that the class of nonpathological functions includes both $C$-regular and semiconcave functions.

Theorem 4.4. Let $V(x)$ be any locally Lipschitz continuous, positive definite, radially unbounded, and nonpathological function. Let $h(x)$ be any continuous, positive definite, and radially unbounded function. Moreover, let $H$ be defined as in (2.2), and assume that

$$
\forall x \in \mathbb{R}^{n}, \quad \exists p_{0} \in \partial_{C} V(x) \quad \text { such that } H\left(x, p_{0}\right)=0 .
$$

Let $\alpha$ and $\gamma$ be given positive numbers, and assume that the following condition holds.

(H) There exists a real constant $R<1$ such that the following inequality holds:

$$
\gamma\left(A_{1}^{2}+\cdots+A_{m}^{2}\right)-2 \alpha\left(A_{1} B_{1}+\cdots+A_{m} B_{m}\right)-R h(x) \leq 0
$$

for each $x \in \mathbb{R}^{n}(x \neq 0)$ and each choice of the real indeterminates $A_{1}, \ldots, A_{m}$ and $B_{1}, \ldots, B_{m}$ subject to the following constraints:

$$
A_{i}, B_{i} \in\left[\underline{D}_{C} V\left(x, g_{i}(x)\right), \bar{D}_{C} V\left(x, g_{i}(x)\right)\right] \quad \text { for } i=1, \ldots, m \text {. }
$$

Then, the feedback law (1.5) Filippov stabilizes system (1.1).

Proof. As in the proof of Theorem 4.1, we will apply Proposition A.4. Let $a \in$ $\dot{\bar{V}}^{(4.13)}(x)$. By construction, there exists $\bar{q} \in \partial_{C} V(x)$ such that, for each $p \in$ $\partial_{C} V(x)$, we have

$$
a=p \cdot f(x)-\alpha(\bar{q} G(x))(p G(x))^{\mathbf{t}}
$$

In order to prove the theorem, it is therefore sufficient to show the following claim.

Claim 1. For each $x \neq 0$, there exists $p_{0} \in \partial_{C} V(x)$ such that, for each $q \in \partial_{C} V(x)$,

$$
p_{0} \cdot f(x)-\alpha(q G(x))\left(p_{0} G(x)\right)^{\mathbf{t}}<0
$$


Let $p_{0}$ be as in (4.14) and let $q$ be any element in $\partial_{C} V(x)$. We have

$$
\begin{aligned}
p_{0} \cdot f(x)-\alpha(q G(x))\left(p_{0} G(x)\right)^{\mathbf{t}} & \\
& =\frac{1}{2}\left[-h(x)+\left(\gamma\left(p_{0} G(x)\right)\left(p_{0} G(x)\right)^{\mathbf{t}}-2 \alpha(q G(x))\left(p_{0} G(x)\right)^{\mathbf{t}}\right)\right] .
\end{aligned}
$$

For each $x \neq 0$, we interpret $A_{1}, \ldots, A_{n}$ as the components of the vector $p_{0} G(x)$ and, respectively, $B_{1}, \ldots, B_{n}$ as the components of the vector $q G(x)$. Now, (4.16) is fulfilled and (4.15) is applicable so that we finally have

$$
p_{0} \cdot f(x)-\alpha(q G(x))\left(p_{0} G(x)\right)^{\mathbf{t}} \leq \frac{h(x)}{2}(R-1)<0
$$

Taking into account Proposition 2.1, we immediately have the following corollary.

Corollary 4.5. Let $h$ be positive definite, continuous, and radially unbounded. Assume that the optimal regulation problem is solvable and that the value function $V$ is locally Lipschitz continuous, nonpathological, and radially unbounded. Assume finally condition (H). Then, the feedback law (1.5) Filippov stabilizes system (1.1).

In order to grasp the meaning of condition $(\mathrm{H})$, we focus on the single-input case $(m=1)$. Writing $A, B$ instead of $A_{1}, B_{1}$, conditions (4.15), (4.16) reduce to

$$
\gamma A^{2}-2 \alpha A B-R h(x) \leq 0
$$

for each $x \in \mathbb{R}^{n}(x \neq 0)$ and each choice of the pair $A, B$ satisfying

$$
A, B \in\left[\underline{D}_{C} V(x, g(x)), \bar{D}_{C} V(x, g(x))\right] .
$$

In the plane of coordinates $A, B,(4.21)$ defines a region bounded by the branches of a hyperbola. Our assumptions amount to say that the square

$$
Q=\left[\underline{D}_{C} V(x, g(x)), \bar{D}_{C} V(x, g(x))\right] \times\left[\underline{D}_{C} V(x, g(x)), \bar{D}_{C} V(x, g(x))\right]
$$

is contained in this region, which means that the distance between $\underline{D}_{C} V(x, g(x))$ and $\bar{D}_{C} V(x, g(x))$ should not be too large. Note that the "north-east" and the "south-west" corners of $Q$ lie on the line $B=A$.

In order to rewrite the condition in a more explicit way, we distinguish several cases. From now on we set for simplicity $\underline{D}=\underline{D}_{C} V(x, g(x))$ and $\bar{D}=\bar{D}_{C} V(x, g(x))$. 
1172 Nonsmooth optimal regulation and discontinuous stabilization

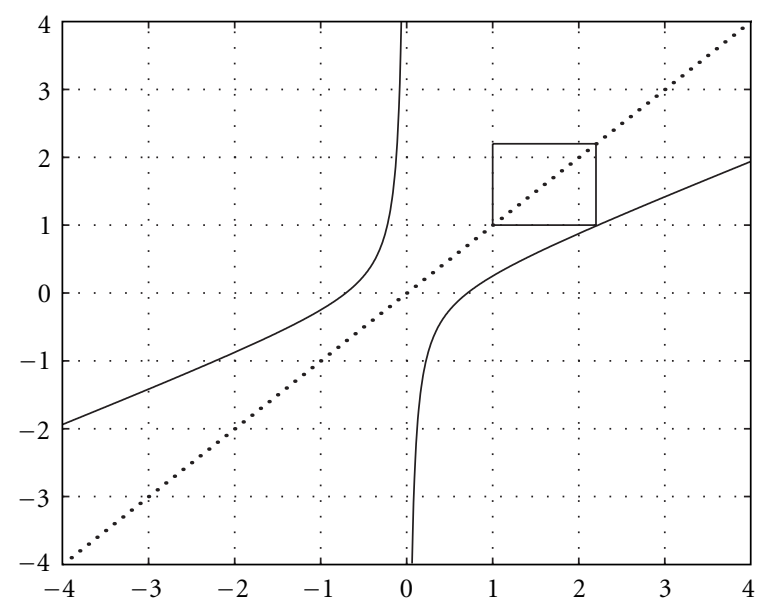

Figure 4.1. First case: $0<R<1, \gamma \leq 2 \alpha$.

First case. Assume that conditions (4.21), (4.22) are verified with $0<R<1$, and let $\gamma \leq 2 \alpha$. The line $B=A$ is contained in the "good" region (see Figure 4.1). Let

$$
A_{0}=\sqrt{\frac{R h(x)}{\gamma+2 \alpha}}
$$

be the abscissa of the intersection between the line $B=-A$ and the right branch of the hyperbola. Then, conditions (4.21), (4.22) are equivalent to

$$
\underline{D} \geq \begin{cases}\frac{\gamma \bar{D}^{2}-R h(x)}{2 \alpha \bar{D}}, & \text { if } \bar{D} \geq A_{0}, \\ \frac{\alpha \bar{D}-\sqrt{\alpha^{2} \bar{D}^{2}+\gamma R h(x)}}{\gamma}, & \text { if } \bar{D} \leq A_{0}\end{cases}
$$

(for $\bar{D}=A_{0}$, the two formulas coincide).

When $\gamma>2 \alpha$, the line $B=A$ crosses the hyperbola in two points whose abscissas are $A_{1}=\sqrt{R h(x) /(\gamma-2 \alpha)}$ and $-A_{1}$ (see Figure 4.2). Conditions (4.21), (4.22) are still reducible to (4.25), but it can be satisfied only if

$$
\bar{D} \leq A_{0} \quad \text { or } \quad \underline{D} \geq-A_{0} .
$$

Second case. Assume now that conditions (4.21), (4.22) are verified with $R=0$. In this case, the hyperbola degenerates and the "good" region becomes a cone. It contains the line $B=A$ if and only if $\gamma \leq 2 \alpha$. Hence, the condition is never satisfied if $\gamma>2 \alpha$.

If $\gamma=2 \alpha$, the condition is satisfied provided that $\underline{D}=\bar{D}$, and hence, in particular when $V$ is smooth. 
A. Bacciotti and F. Ceragioli 1173

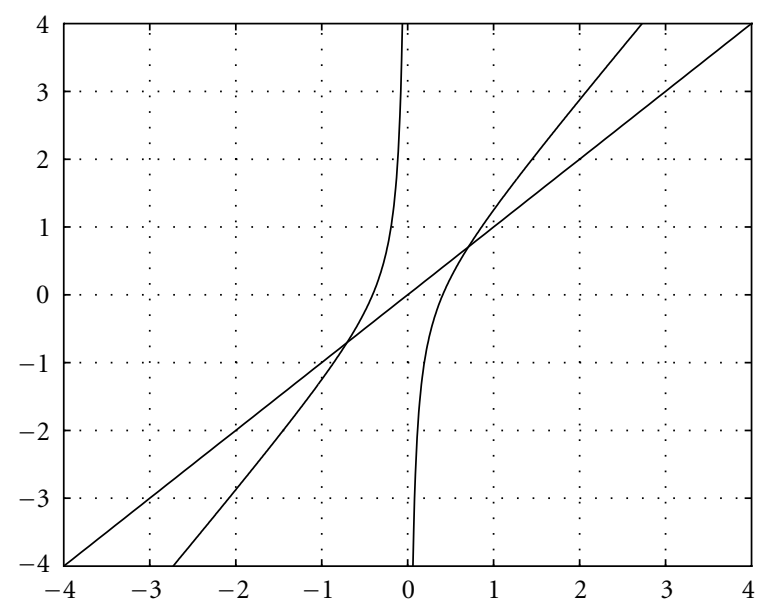

Figure 4.2. First case: $0<R<1, \gamma>2 \alpha$.

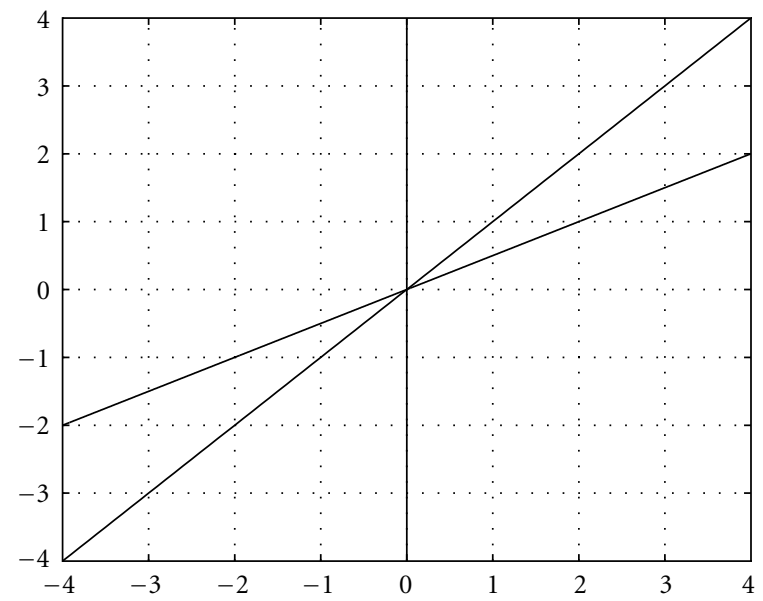

Figure 4.3. Second case: $R=0, \gamma<2 \alpha$.

Finally, if $\gamma<2 \alpha$, conditions (4.25) simplify in the following manner (see Figure 4.3):

$$
\underline{D} \geq \begin{cases}\frac{\gamma \bar{D}}{2 \alpha}, & \text { if } \bar{D} \geq 0, \\ \frac{2 \alpha \bar{D}}{\gamma}, & \text { if } \bar{D}<0 .\end{cases}
$$


1174 Nonsmooth optimal regulation and discontinuous stabilization

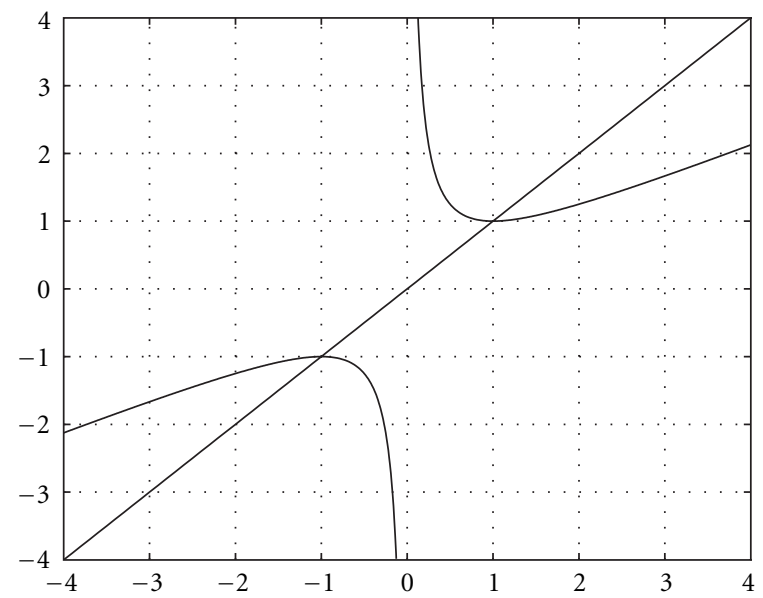

Figure 4.4. Third case: $R<0, \gamma<2 \alpha$.

Third case. Assume finally that conditions (4.21), (4.22) are verified with $R<0$. The "good" regions are now the convex regions bounded by the branches of the hyperbola (see Figure 4.4).

The conditions are never satisfied if $\gamma \geq 2 \alpha$. For $\gamma<2 \alpha$, the conditions are given by (4.25). However, the conditions cannot be satisfied if

$$
0 \leq \bar{D}<A_{1} \quad \text { or } \quad-A_{1}<\underline{D} \leq 0 .
$$

Remark 4.6. Note that in certain cases stabilization is possible even if $2 \alpha<\gamma$ (typically, this happens for stabilizable driftless systems).

\section{Sufficient conditions for optimality}

In this section, we enlarge the class of admissible inputs to all measurable, locally bounded maps $u(t):[0,+\infty) \rightarrow \mathbb{R}^{m}$. The aim is to extend the following result, whose proof can be found in $[8,25,33]$ in slightly different forms.

Optimality principle. If the Hamilton-Jacobi equation (2.5) admits a positive definite $C^{1}$-solution $V(x)$ such that $V(0)=0$, and if the feedback (1.5) with $\alpha=\gamma$ is a global stabilizer for (1.1), then, for each initial state $x$, trajectories corresponding to the same feedback law minimize the cost functional (1.3) over all the admissible inputs $u(t)$ for which $\lim _{t \rightarrow+\infty} \varphi(t ; x, u(\cdot))=0$. Moreover, $V(x)$ coincides with the value function.

As remarked in [33], restricting the minimization to those inputs whose corresponding solutions converge to zero can be interpreted as incorporating a detectability condition. In this section, we make the detectability condition explicit by assuming that $h$ is positive definite. 
The following result can be seen as a partial converse of Proposition 2.1. Roughly speaking, it says that if the closed loop system admits a Carathéodory solution satisfying the necessary conditions and driving the system asymptotically to zero, then this solution is optimal.

Theorem 5.1. Consider the optimal regulation problem (1.3) with $h(x)$ continuous, positive definite, and radially unbounded, and let $V(x)$ be any locally Lipschitz continuous, radially unbounded, and positive definite function. Assume in addition that $V(x)$ is nonpathological. Let $H$ be defined according to (2.2), and assume that

(A) for all $x \in \mathbb{R}^{n}$ and for all $p \in \partial_{C} V(x), H(x, p) \leq 0$.

Let $x^{o} \in \mathbb{R}^{n}$, and let $u^{o}(t)$ be any admissible input. For simplicity, write $\varphi^{o}(t)=$ $\varphi\left(t ; x^{o}, u^{o}(\cdot)\right)$ and assume that

(B) for a.e. $t \geq 0$, there exists $p^{o}(t) \in \partial_{C} V\left(\varphi^{o}(t)\right)$ such that

(i) $H\left(\varphi^{o}(t), p^{o}(t)\right)=0$,

(ii) $u^{o}(t)=-\gamma\left(p^{o}(t) G\left(\varphi^{o}(t)\right)\right)^{\mathrm{t}}$;

(C) $\lim _{t \rightarrow+\infty} \varphi^{o}(t)=0$.

Then, $u^{o}(t)$ is optimal for $x^{o}$. Moreover, the value function of the optimal regulation problem and $V(x)$ coincides at $x^{o}$.

Proof. Since $\varphi^{o}(t)$ is absolutely continuous, by (B)(ii) we have, for a.e. $t \geq 0$,

$$
\begin{aligned}
\dot{\varphi}^{o}(t) & =f\left(\varphi^{o}(t)\right)-G\left(\varphi^{o}(t)\right) u^{o}(t) \\
& =f\left(\varphi^{o}(t)\right)-\gamma G\left(\varphi^{o}(t)\right)\left(p^{o}(t) G\left(\varphi^{o}(t)\right)\right)^{\mathbf{t}} .
\end{aligned}
$$

Using (B)(i), we can now compute the cost

$$
\begin{aligned}
J\left(x^{o}, u^{o}(\cdot)\right) & =\frac{1}{2} \int_{0}^{+\infty}\left(h\left(\varphi^{o}(t)\right)+\frac{\left|u^{o}(t)\right|^{2}}{\gamma}\right) d t \\
& =\int_{0}^{+\infty}-p^{o}(t)\left[f\left(\varphi^{o}(t)\right)-\gamma G\left(\varphi^{o}(t)\right) u^{o}(t)\right] d t \\
& =\int_{0}^{+\infty}-p^{o}(t) \dot{\varphi}^{o}(t) d t=V\left(x^{o}\right)
\end{aligned}
$$

where the last equality follows by virtue of Lemma B.6 and (C). In order to complete the proof, we now show that, for any other admissible input $u(t)$, we have

$$
V\left(x^{o}\right)=J\left(x^{o}, u^{o}(\cdot)\right) \leq J\left(x^{o}, u(\cdot)\right) .
$$

For simplicity, we use again a shortened notation $\varphi(t)=\varphi\left(t ; x^{o}, u(\cdot)\right)$. We distinguish two cases.

(1) The integral in (1.3) diverges. In this case, it is obvious that $J\left(x^{o}, u^{o}(\cdot)\right)=$ $V\left(x^{o}\right)<J\left(x^{o}, u(\cdot)\right)$.

(2) The integral in (1.3) converges. According to Lemma B.5, we conclude that $\lim _{t \rightarrow+\infty} \varphi(t)=0$, and since $V(x)$ is radially unbounded, continuous, and 
positive definite, this in turn implies $\lim _{t \rightarrow+\infty} V(\varphi(t))=0$. Let $p(t)$ be any measurable selection of the set-valued map $\partial_{C} V(\varphi(t))$ (such a selection exists since $\partial_{C} V(\varphi(t))$, the composition of an upper semicontinuous set-valued map and a continuous single-valued map, is upper semicontinuous, hence measurable; see [4]). By (A), and the usual "completing the square" method, we have

$$
\begin{aligned}
J\left(x^{o}, u(\cdot)\right)= & \frac{1}{2} \int_{0}^{+\infty}\left(h(\varphi(t))+\frac{|u(t)|^{2}}{\gamma}\right) d t \\
\geq & \int_{0}^{+\infty}\left[-p(t) f(\varphi(t))+\frac{\gamma}{2}|p(t) G(\varphi(t))|^{2}+\frac{|u(t)|^{2}}{2 \gamma}\right] d t \\
= & \int_{0}^{+\infty}[-p(t) f(\varphi(t))-p(t) G(\varphi(t)) u(t) \\
& \left.\quad+\frac{1}{2 \gamma}|\gamma p(t) G(\varphi(t))+u(t)|^{2}\right] d t \\
\geq & \int_{0}^{+\infty}-p(t) \dot{\varphi}(t) d t=V\left(x^{o}\right),
\end{aligned}
$$

where we used again Lemma B.6. This achieves the proof. In particular, we see that $u^{o}(t)$ is optimal, and we see that the value function of the minimization problem (1.3) coincides with $V(x)$ at $x^{o}$.

Note that (C) is actually needed since $h(x)$ is positive definite (see Lemma B.5). It could be replaced by the assumption that $J\left(x^{o}, u^{o}(\cdot)\right)$ is finite.

Corollary 5.2. Let $h(x)$ be continuous, radially unbounded, and positive definite. Let $V(x)$ be any locally Lipschitz continuous, radially unbounded, and positive definite function. Assume in addition that $V(x)$ is nonpathological. Finally, let $H$ be defined according to (2.2), and assume that (4.2) holds. Then, for each $x \in \mathbb{R}^{n}$, there exists a measurable, locally bounded control which is optimal for the minimization problem (1.3). Moreover, the value function and $V(x)$ coincide at every $x \in \mathbb{R}^{n}$.

Proof. Let $x^{0} \in \mathbb{R}^{n}$ and let $\varphi^{o}(t)$ be any solution of the initial value problem

$$
\dot{x} \in f(x)+G(x) k_{y}(x), \quad x(0)=x^{o},
$$

where for a.e. $x \in \mathbb{R}^{n}, k_{\gamma}(x)=-\gamma(\nabla V(x) G(x))^{\mathbf{t}}$ (i.e., at those points where the gradient exists, $k_{\gamma}$ is given by (1.5) with $\alpha=\gamma$ ). By virtue of Theorem 4.1, we can assume that $k_{\gamma}(x)$ is continuous so that such a $\varphi^{o}(t)$ exists, and it is a solution in the classical sense. From the proof of Theorem 4.1, it is also clear that $k_{\gamma}(x)=$ $-\gamma(p G(x))^{\mathbf{t}}$ for each $p \in \partial_{C} V(x)$ and each $x \in \mathbb{R}^{n}$. Since $x \mapsto \partial_{C} V(x)$ is compact convex valued and upper semicontinuous, by Filippov's lemma (see [4]), there exists a measurable map $p^{o}(t) \in \partial_{C} V\left(\varphi^{o}(t)\right)$ such that for a.e. $t \geq 0$, one has $k_{\gamma}\left(\varphi^{o}(t)\right)=-\gamma\left(p^{o}(t) G\left(\varphi^{o}(t)\right)\right)^{\mathbf{t}}$. 


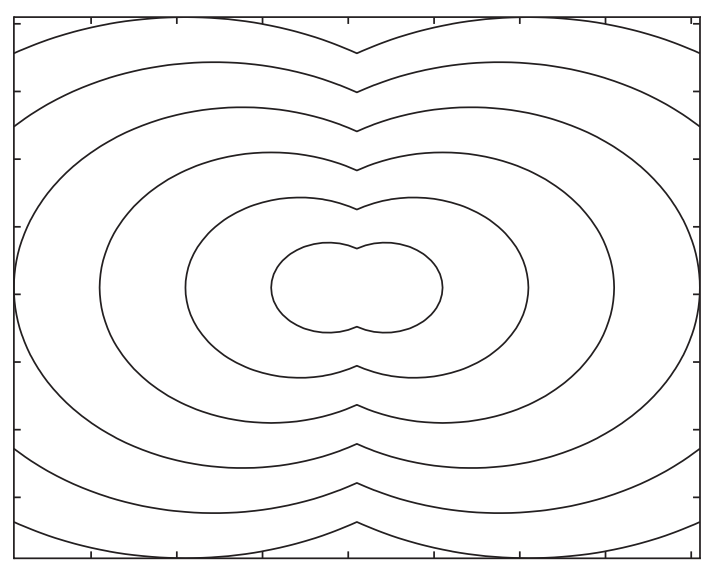

Figure 6.1. Level curves of $V(x, y)=\left(4 x^{2}+3 y^{2}\right)^{1 / 2}-|x|$.

We set $u^{o}(t)=-\gamma\left(p^{o}(t) G\left(\varphi^{o}(t)\right)\right)^{\mathrm{t}}$. Note that $\varphi^{o}(t)$ is the unique solution of (1.1) issuing from $x^{o}$ and corresponding to the admissible input $u^{o}(t)$.

Theorem 4.1 also states that $k_{\gamma}(t)$ is a stabilizing feedback. In conclusion, all the assumptions (A), (B), and (C) of Theorem 5.1 are fulfilled. The statement is proven.

\section{Examples}

The results of the previous sections are illustrated by the following examples.

Example 6.1. Consider the two-dimensional, single-input driftless system

$$
\left(\begin{array}{c}
\dot{x} \\
\dot{y}
\end{array}\right)=u g(x, y), \quad g(x, y)=\left(\begin{array}{c}
x^{2}-y^{2} \\
2 x y
\end{array}\right)
$$

(the so-called Artstein's circles example). The function $V(x, y)=\sqrt{4 x^{2}+3 y^{2}}-$ $|x|$ is a control Lyapunov function (in the sense of proximal gradient) for this system. As a sum of a function of class $C^{1}$ and a concave function, $V$ is semiconcave in $\mathbb{R}^{2} \backslash\{(0,0)\}$, but not differentiable when $x=0$ (the level curves are piecewise arcs of circumferences, see Figure 6.1).

We want to construct an optimization problem with $\gamma=1$, whose value function is $V$. To this purpose, we follow an "inverse optimality" approach (see [33]). Define

$$
h(x, y)= \begin{cases}{\left[\frac{x}{\sqrt{4 x^{2}+3 y^{2}}}\left(4 x^{2}+2 y^{2}-|x| \sqrt{4 x^{2}+3 y^{2}}\right)+(\operatorname{sgn} x) y^{2}\right]^{2},} & \text { if } x \neq 0, \\ y^{4}, & \text { if } x=0 .\end{cases}
$$



form

Note that $h(x, y)$ is continuous and positive definite. Equation (2.5) takes the

$$
(\nabla V(x, y) g(x, y))^{2}=h(x, y)
$$

A simple computation shows that it is fulfilled in the usual sense if $x \neq 0$. In points where $x=0$, we have

$$
\partial_{C} V(0, y)=\left(p_{1}, \sqrt{3} \operatorname{sgn} y\right), \quad p_{1} \in[-1,1]
$$

and the Hamilton-Jacobi equation reduces to $(\partial V / \partial x)^{2}=1$. Consistently with Propositions 2.1 and 2.3, we therefore see that $V$ is a viscosity subsolution, and actually a viscosity solution (note that the subdifferential is empty for $x=0$, $y \neq 0$ ), as well as a solution in extended sense. We also see that $V$ is a viscosity supersolution of (2.6) but not a viscosity subsolution of such equation.

The damping feedback $k_{1}(x)$ corresponding to $V$ (i.e., (1.5) with $\alpha=1$ ) is easily computed for $x \neq 0$ (it coincides with minus the expression inside the square brackets in (6.2)). It turns out to be positive if $x<0$ and negative if $x>0$. It is discontinuous along the $y$-axis. Its construction can be completed in such a way that condition (i) of Proposition 2.1 is preserved. In fact, at the points of the form $(0, y)$, there are two possible choices of the vector $p_{0}$. Both of them give rise to a stabilizing feedback provided that solutions are intended in Carathéodory sense.

Now let $\varphi^{o}(t)$ be any Carathéodory solution of the closed loop system, and let $u^{o}(t)=k_{1}\left(\varphi^{o}(t)\right)$. The assumptions (A), (B), and (C) of Theorem 5.1 are fulfilled. Thus, all the solutions of the closed loop system are optimal and $V$ is actually the value function.

Note that in this example optimal controls are not unique. Note also that the damping feedback does not stabilize the system in Filippov sense. On the other hand, it is well known that Artstein's circles system cannot be stabilized in Filippov sense.

Example 6.2. Given the two-dimensional, single-input linear system

$$
\dot{x}=-x, \quad \dot{y}=y+2 u,
$$

we want to impose the value function $V(x, y)=|x|+y^{2}$. Note that $V$ is $C$ regular, but not differentiable for $x=0$. Its level curves are plotted in Figure 6.2. We set

$$
h(x, y)=2|x|+12 y^{2}
$$

and $\gamma=1$. The function $h(x)$ is continuous, positive definite, and radially unbounded. In points where $x \neq 0, V$ is smooth: the Hamilton-Jacobi equation is 


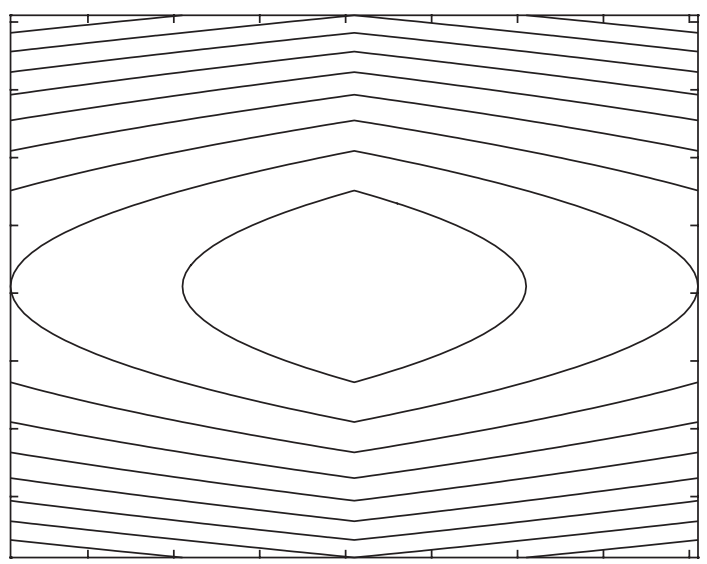

Figure 6.2. Level curves of $V(x, y)=|x|+y^{2}$.

fulfilled in the usual sense. In points where $x=0$, we have

$$
\partial_{C} V(0, y)=\left(p_{1}, 2 y\right), \quad p_{1} \in[-1,1]
$$

The Hamilton-Jacobi equation reduces to an identity in these points, so that (4.2) is satisfied. According to Theorems 4.1 and 5.1, the damping feedback is continuous. It takes the form

$$
k_{\alpha}(x, y)=-\alpha \nabla V(x, y) g(x, y)=-4 \alpha y .
$$

Hence, it is a stabilizer for $\alpha \geq 1 / 2$ (actually, we have a larger stability margin: $\alpha>1 / 8$ ). Moreover, for $\alpha=1$, it generates the optimal solutions. Finally, thanks to Corollary 5.2, $V(x)$ coincides with the value function.

Note that in this example matrix $G$ is constant. Nevertheless, in points of the form $(0, y)$, the Hamiltonian function $H$ is not strictly convex with respect to $p$. Example 6.3. First we consider the single-input driftless system

$$
\left(\begin{array}{l}
\dot{x} \\
\dot{y}
\end{array}\right)=u g(x, y), \quad g(x, y)=\left(\begin{array}{l}
x \\
y
\end{array}\right),
$$

and choose the semiconcave function $V$ of Example 6.1. To interpret $V$ as a value function, we define $\gamma=1$ and $h(x, y)=V^{2}(x, y)$. Theorem 4.1 is applicable and the damping feedback law is continuous. Optimality of solutions and the fact that $V$ is the value function are guaranteed by Corollary 5.2. Similar conclusions are obtained if we take the semiconvex function $V(x, y)=\sqrt{4 x^{2}+3 y^{2}}+|x|$.

Finally, we consider system (6.9) and the associated optimal regulation problem with $y=1$ and $h(x, y)=\left((7 / 2) x^{2}+(13 / 2) y^{2}-3 \sqrt{3}|x| y\right)^{2}$. The value function in this case is given by $V(x, y)=(7 / 4) x^{2}+(13 / 4) y^{2}-(3 \sqrt{3} / 2)|x| y$. Such 


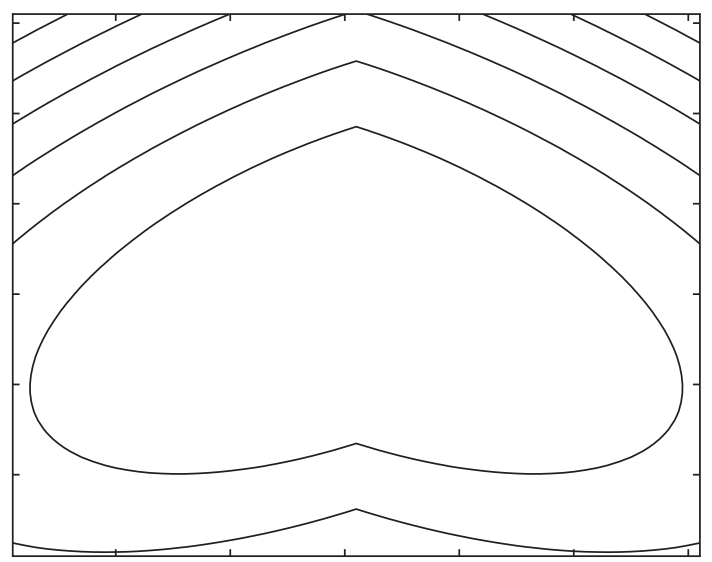

Figure 6.3. Level curves of $V(x, y)=(7 / 4) x^{2}+(13 / 4) y^{2}-\left(3^{3 / 2} / 2\right)|x| y$.

a function $V$ is neither $C$-regular nor semiconcave, but it is nonpathological (the levels curves are plotted in Figure 6.3). Even in this case, Theorem 4.1 and Corollary 5.2 are applicable.

Example 6.4. In this example, we consider the system

$$
\dot{x}=u, \quad \dot{y}=-y^{3}
$$

and the function $V(x, y)=x^{2}+y^{2}+|x| y^{2}$ (see Figure 6.4). By direct computation, it is possible to see that $\partial_{C} V(x, y)=\underline{\partial} V(x, y)$ at each point so that $V$ is $C$-regular, and hence, nonpathological. In particular, along the $y$-axis, we have

$$
\partial_{C} V(0, y)=\left(p_{1}, 2 y\right), \quad p_{1} \in\left[-y^{2}, y^{2}\right] \text {. }
$$

Define $\gamma=1$ and $h(x, y)=4 x^{2}+5 y^{2}+4|x|\left(y^{4}+y^{2}\right)$. Then the HamiltonJacobi equation (2.5) is satisfied by $V(x)$ in the usual sense when $x \neq 0$. Along the $y$-axis, the Hamiltonian reduces to $(1 / 2)\left(p_{1}^{2}-y^{4}\right)$. Thus, we have $H(p, 0, y) \leq 0$ for each $p$ in the Clarke gradient, but the equality holds only at the extremal points. Hence, $V(x, y)$ is seen to be a viscosity subsolution of (2.5) (since $V$ is $C$-regular but not differentiable along the $y$-axis, the superdifferential is empty in these points), but not a supersolution.

In particular, condition (4.2) is not met and Theorem 4.1 is not applicable. Nevertheless, the system is Filippov stabilized by the (discontinuous) damping feedback $k_{1}(x, y)=-2 x-(\operatorname{sgn} x) y^{2}$. Indeed, a simple computation shows that condition $(\mathrm{H})$ is fulfilled with $3 / 5 \leq R<1$ so that we can use Theorem 4.4.

As far as the existence of optimal controls is concerned, we make the following important remark. Given an initial point $(\bar{x}, \bar{y})$, we do not have for sure that there is a Carathéodory solution of the closed loop system issuing from $(\bar{x}, \bar{y})$ 


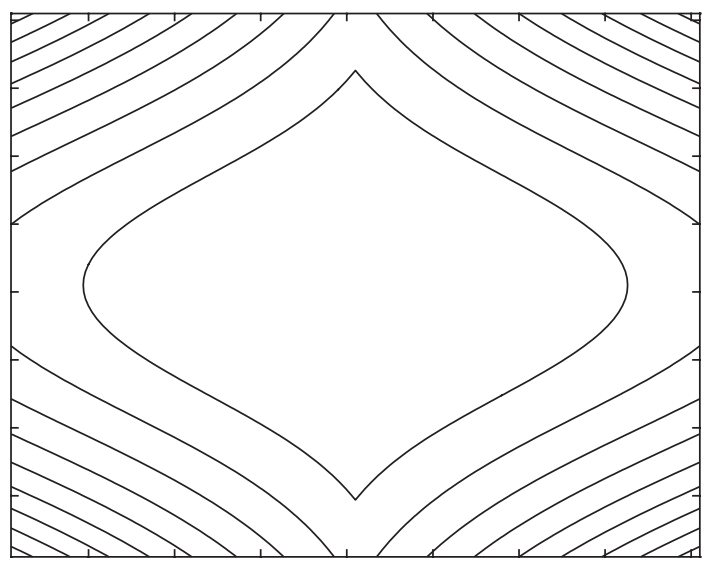

Figure 6.4. Level curves of $V(x, y)=x^{2}+y^{2}+|x| y^{2}$.

and asymptotically going to the origin. In fact, by numerical simulation, one realizes that this is actually false, with the exception of the points along the $x$ axis. As a matter of fact, if $\bar{x} \neq 0, \bar{y} \neq 0$, the solution starting from $(\bar{x}, \bar{y})$ hits the $y$-axis at some point $(0, \hat{y})$ with $\hat{y} \neq 0$. The only way to construct a Carathéodory solution issuing from a point $(0, \bar{y})$, moving along the $y$-axis, and asymptotically going to the origin, is taking $u=0$. But in this way the necessary conditions for optimality fail. In fact, by direct computation, it is possible to see that the cost of such a solution is strictly greater than $V(0, \bar{y})$.

In conclusion, according to the theory developed in this paper, we have the following alternative: either $V$ is not the value function of the optimal regulation problem or there exist no optimal controls (with the exceptions of points along the $x$-axis).

Example 6.5. Consider the two-dimensional driftless system with two inputs

$$
\left(\begin{array}{c}
\dot{x} \\
\dot{y}
\end{array}\right)=u_{1} g_{1}(x, y)+u_{2} g_{2}(x, y), \quad g_{1}(x, y)=\left(\begin{array}{c}
x+y \\
x+y
\end{array}\right), g_{2}(x, y)=\left(\begin{array}{c}
x-y \\
-x+y
\end{array}\right) \text {. }
$$

In order to impose the value function $V(x, y)=|x|+|y|$, we try $h(x, y)=$ $4(|x|+|y|)^{2}$. Note that $V$ is locally Lipschitz continuous and $C$-regular, while $h$ is continuous and positive definite. As before, we set $\gamma=1$. For $x y \neq 0, V$ is differentiable, and the Hamilton-Jacobi equation is satisfied in the usual sense. This allows us to construct a (discontinuous) feedback in the damping form

$$
\begin{aligned}
& u_{1}=k_{1}(x, y)=-2(|x|+|y|), \quad u_{2}=k_{2}(x, y)=0 \quad \text { if } x y>0, \\
& u_{1}=k_{1}(x, y)=0, \quad u_{2}=k_{2}(x, y)=-2(|x|+|y|) \quad \text { if } x y<0 .
\end{aligned}
$$


In points of the form $(x, 0)$, we have

$$
\partial_{C} V(x, 0)=\left(1, p_{2}\right), \quad p_{2} \in[-1,1],
$$

so that the Hamilton-Jacobi equation reduces to

$$
2 x^{2}\left(1+p_{2}^{2}\right)=4 x^{2} .
$$

This equality is satisfied only for $p_{2}= \pm 1$. Even in this case, we see that $V$ is a viscosity subsolution of (2.5), but not a supersolution. Unfortunately, if we complete the construction of the feedback (6.13) according to one of these choices, the closed loop system has no (Carathéodory) solution issuing from $(x, 0)$. In fact, the only way to construct a solution going to the origin for $t \rightarrow+\infty$ is to take a strict convex combination of the two vector fields $g_{1}, g_{2}$, but this cannot be done in "optimal" way.

In conclusion, the necessary conditions are not satisfied for points of the form $(x, 0)$ so that we have again this alternative: either $V$ is not the value function or the optimal regulation problem is not solvable for these points. Actually, we conjecture that in this example the optimal regulation problem is solvable only for points lying on the lines $y= \pm x$.

\section{Appendices}

\section{A. Tools from nonsmooth analysis}

For reader's convenience, we shortly review the definitions of the various extensions of derivatives and gradients used in this paper (see $[13,16]$ as general references). Moreover, we prove two apparently new results on Clarke regular and semiconvex functions.

Given a function $V: \mathbb{R}^{n} \rightarrow \mathbb{R}$, for any $x, v \in \mathbb{R}^{n}$ and for any $h \in \mathbb{R} \backslash\{0\}$, we consider the difference quotient

$$
\mathscr{R}(h, x, v)=\frac{V(x+h v)-V(x)}{h} .
$$

If there exists $\lim _{h \rightarrow 0^{+}} \mathscr{R}(h, x, v)$, then it is called the directional derivative of $V$ at $x$ in the direction $v$ and is denoted by $D^{+} V(x, v)$.

When $V$ does not admit directional derivative in the direction $v$, we may substitute this notion with a number of different generalizations. We limit ourselves to the definitions we use in this paper.

The so-called Dini directional derivatives associate to each $x$ four numbers $\underline{D}^{+} V(x, v), \bar{D}^{+} V(x, v), \underline{D}^{-} V(x, v)$, and $\bar{D}^{-} V(x, v)$, where the former is defined as

$$
\underline{D}^{+} V(x, v)=\liminf _{h \rightarrow 0^{+}} \mathscr{R}(h, x, v),
$$

and the others are analogously defined. When $\underline{D}^{+} V(x, v)=\underline{D}^{-} V(x, v)$ (resp., $\left.\bar{D}^{+} V(x, v)=\bar{D}^{-} V(x, v)\right)$, we simply write it as $\underline{D} V(x, v)($ resp., $\bar{D} V(x, v))$. 
If we let $v$ vary as well, we get the so-called contingent directional derivatives $\underline{D}_{K}^{+} V(x, v), \bar{D}_{K}^{+} V(x, v), \underline{D}_{K}^{-} V(x, v)$, and $\bar{D}_{K}^{-} V(x, v)$. More precisely, the lower right contingent directional derivative is defined as

$$
\underline{D}_{K}^{+} V(x, v)=\liminf _{h \rightarrow 0^{+}, w \rightarrow v} \mathscr{R}(h, x, w),
$$

and the others are defined in a similar way. When $V$ is locally Lipschitz continuous, Dini derivatives and contingent derivatives coincide.

Clarke introduced another kind of generalized directional derivative by letting $x$ vary:

$$
\bar{D}_{C} V(x, v)=\limsup _{h \rightarrow 0, y \rightarrow x} \mathscr{R}(h, y, v), \quad \underline{D}_{C} V(x, v)=\liminf _{h \rightarrow 0, y \rightarrow x} \mathscr{R}(h, y, v) .
$$

Besides directional derivatives, different generalizations of the differential have been defined in the literature.

The subdifferential can be seen as a generalization of Fréchet differential:

$$
\underline{\partial} V(x)=\left\{p \in \mathbb{R}^{n}: \liminf _{h \rightarrow 0} \frac{V(x+h)-V(x)-p \cdot h}{|h|} \geq 0\right\} .
$$

Analogously, the superdifferential is defined as

$$
\bar{\partial} V(x)=\left\{p \in \mathbb{R}^{n}: \limsup _{h \rightarrow 0} \frac{V(x+h)-V(x)-p \cdot h}{|h|} \leq 0\right\} .
$$

These objects can be used in order to define the notions of viscosity superand subsolutions of partial differential equations of the Hamilton-Jacobi type (see $[7,16])$. The sub- and superdifferentials can be characterized by means of contingent derivatives (see [21]). Indeed, we have

$$
\begin{aligned}
& \underline{\partial} V(x)=\left\{p \in \mathbb{R}^{n}: p \cdot v \leq \underline{D}_{K}^{+} V(x, v) \forall v \in \mathbb{R}^{n}\right\}, \\
& \bar{\partial} V(x)=\left\{p \in \mathbb{R}^{n}: p \cdot v \geq \bar{D}_{K}^{+} V(x, v) \forall v \in \mathbb{R}^{n}\right\} .
\end{aligned}
$$

For each $x, \underline{\partial} V(x)$ (and analogously $\bar{\partial} V(x))$ is a convex and closed set, and it may be empty. If $V$ is differentiable at $x$, then it coincides with the singleton $\{\nabla V(x)\}$.

The proximal subdifferential is defined as

$$
\begin{gathered}
\partial_{P} V(x)=\left\{p \in \mathbb{R}^{n}: \exists \sigma \geq 0, \exists \delta \geq 0 \text { such that }\left(z \in \mathbb{R}^{n},|z-x|<\delta v\right)\right. \\
\left.\Longrightarrow\left(V(z)-V(x) \geq p \cdot(z-x)-\sigma|z-x|^{2}\right)\right\} .
\end{gathered}
$$

For each $x, \partial_{P} V(x)$ is convex, but not necessarily closed. Moreover, $\partial_{P} V(x) \subseteq$ $\underline{\partial} V(x)$.

The Clarke generalized gradient is defined as

$$
\partial_{C} V(x)=\left\{p \in \mathbb{R}^{n}: p \cdot v \leq \bar{D}_{C} V(x, v), \forall v \in \mathbb{R}^{n}\right\}
$$


1184 Nonsmooth optimal regulation and discontinuous stabilization

or, equivalently,

$$
\partial_{C} V(x)=\left\{p \in \mathbb{R}^{n}: p \cdot v \geq \underline{D}_{C} V(x, v), \forall v \in \mathbb{R}^{n}\right\} .
$$

It is possible to see that $\bar{D}_{C} V(x, v)=\sup \left\{p \cdot v: p \in \partial_{C} V(x)\right\}$ and $\underline{D}_{C} V(x, v)=$ $\inf \left\{p \cdot v: p \in \partial_{C} V(x)\right\}$.

If $V$ is Lipschitz continuous, by Rademacher's theorem, its gradient $\nabla V(x)$ exists almost everywhere. Let $N$ be the subset of $\mathbb{R}^{n}$ where the gradient does not exist. It is possible to characterize Clarke generalized gradient as

$$
\partial_{C} V(x)=\operatorname{co}\left\{\lim _{x_{i} \rightarrow x} \nabla V\left(x_{i}\right), x_{i} \longrightarrow x, x_{i} \notin N \cup \Omega\right\}
$$

where $\Omega$ is any null measure set. By using this characterization, it is obvious that $\partial_{C} V(x)$ is convex; it is possible to see that it is also compact. Moreover, we have

$$
\partial_{P} V(x) \subseteq \underline{\partial} V(x) \subseteq \partial_{C} V(x)
$$

and also

$$
\bar{\partial} V(x) \subseteq \partial_{C} V(x)
$$

We now give the definition of semiconcave and Clarke regular (briefly $C$ regular) function.

The function $V$ is said to be semiconcave (with linear modulus) if there exists $C>0$ such that

$$
\lambda V(x)+(1-\lambda) V(y)-V(\lambda x+(1-\lambda) y) \leq \lambda(1-\lambda) C|x-y|^{2}
$$

for any pair $x, y \in \mathbb{R}^{n}$ and for any $\lambda \in[0,1]$. Analogously, $V$ is said to be semiconvex if $-V$ is semiconcave.

In the next proposition (see [12]), a few interesting properties of semiconcave functions are collected.

Proposition A.1. If $V$ is semiconcave, then

(i) $V$ is Lipschitz continuous,

(ii) for any $x, v$, there exists $D^{+} V(x, v)$, and $D^{+} V(x, v)=\underline{D}_{C} V(x, v)$,

(iii) $\bar{\partial} V(x)=\partial_{C} V(x)$ for any $x$.

We say that $V$ is $C$-regular if, for all $x, v \in \mathbb{R}^{n}$, there exists $D^{+} V(x, v)$, and $D^{+} V(x, v)=\bar{D}_{C} V(x, v)$.

$C$-regular functions form a rather wide class: for instance, semiconvex functions are $C$-regular. $C$-regular functions can be characterized in terms of generalized gradients in the following way.

Proposition A.2. Let $V: \mathbb{R}^{n} \rightarrow \mathbb{R}$ be locally Lipschitz continuous. $V$ is $C$-regular if and only if $\partial_{C} V(x)=\underline{\partial} V(x)$ for all $x$. 
Proof. We first assume that $V$ is $C$-regular. Since $V$ is also Lipschitz continuous, we have that

$$
\begin{aligned}
\underline{\partial} V(x) & =\left\{p \in \mathbb{R}^{n}: p \cdot v \leq \underline{D}_{K}^{+} V(x, v) \forall v \in \mathbb{R}^{n}\right\} \\
& =\left\{p \in \mathbb{R}^{n}: p \cdot v \leq \underline{D}^{+} V(x, v) \forall v \in \mathbb{R}^{n}\right\} \\
& =\left\{p \in \mathbb{R}^{n}: p \cdot v \leq \bar{D}^{+} V(x, v) \forall v \in \mathbb{R}^{n}\right\} \\
& =\left\{p \in \mathbb{R}^{n}: p \cdot v \leq \bar{D}_{C} V(x, v) \forall v \in \mathbb{R}^{n}\right\} \\
& =\partial_{C} V(x) .
\end{aligned}
$$

We now assume $\partial_{C} V(x)=\underline{\partial} V(x)$ for all $x$. Due to the convexity of $\partial_{C} V(x)$, we get that $\underline{D}_{K}^{+} V(x, v)=\bar{D}_{C} V(x, v)$ for all $x$ and for all $v$. Moreover, we have

$$
\underline{D}_{K}^{+} V(x, v) \leq \underline{D}^{+} V(x, v) \leq \bar{D}^{+} V(x, v) \leq \bar{D}_{C} V(x, v),
$$

which implies that there exists

$$
D^{+} V(x, v)=\bar{D}_{C} V(x, v)
$$

The proofs of Theorems 4.1 and 4.4 rely on the notion of set-valued derivative of a map $V$ with respect to a differential inclusion, introduced in [34] and already exploited in [5]. Given a differential inclusion

$$
\dot{x} \in F(x)
$$

(with $0 \in F(0)$ ), the set-valued derivative of a map $V$ with respect to (A.18) is defined as the closed, bounded (possibly empty) interval

$$
\dot{\bar{V}}^{\text {(A.18) }}(x)=\left\{a \in \mathbb{R}: \exists v \in F(x) \text { such that } p \cdot v=a, \forall p \in \partial_{C} V(x)\right\} .
$$

Such a derivative of the map $V$ can be successfully used in case $V$ is nonpathological in the sense of the following definition given by Valadier in [38].

A function $V$ is said to be nonpathological if for every absolute continuous function $\varphi: \mathbb{R} \rightarrow \mathbb{R}^{n}$ and for a.e. $t$, the set $\partial_{C} V(\varphi(t))$ is a subset of an affine subspace orthogonal to $\dot{\varphi}(t)$.

Note that nonpathological functions form a quite wide class which includes $C$-regular functions. The following proposition can also be easily proven.

Proposition A.3. If $V$ is semiconcave, then it is nonpathological.

Proof. Let $\varphi: \mathbb{R} \rightarrow \mathbb{R}^{n}$ be an absolutely continuous function. Since $V$ is semiconcave, then it is also locally Lipschitz continuous. This implies that $V \circ \varphi$ is absolutely continuous and then, for almost all $t$, there exists $(d / d t) V(\varphi(t))$. Let $t \in \mathbb{R}$ be such that there exist both $\dot{\varphi}(t)$ and $(d / d t) V(\varphi(t))$. Since $V$ is locally 
1186 Nonsmooth optimal regulation and discontinuous stabilization

Lipschitz, we have that

$$
\frac{d}{d t} V(\varphi(t))=\lim _{h \rightarrow 0} \frac{V(\varphi(t)+h \dot{\varphi}(t))-V(\varphi(t))}{h} .
$$

On the one hand, due to Lipschitz continuity of $V$, we have that

$$
\begin{aligned}
\frac{d}{d t} V(\varphi(t)) & =\lim _{h \rightarrow 0^{+}} \frac{V(\varphi(t)+h \dot{\varphi}(t))-V(\varphi(t))}{h} \\
& =D^{+} V(\varphi(t), \dot{\varphi}(t))=\underline{D}_{C} V(\varphi(t), \dot{\varphi}(t)) \\
& =\min \left\{p \cdot \dot{\varphi}(t), p \in \partial_{C} V(\varphi(t))\right\} .
\end{aligned}
$$

On the other hand,

$$
\begin{aligned}
\frac{d}{d t} V(\varphi(t)) & =\lim _{h \rightarrow 0^{-}} \frac{V(\varphi(t)+h \dot{\varphi}(t))-V(\varphi(t))}{h} \\
& =-\lim _{h \rightarrow 0^{+}} \frac{V(\varphi(t)+h(-\dot{\varphi}(t)))-V(\varphi(t))}{h} \\
& =-D^{+} V(\varphi(t),-\dot{\varphi}(t))=-\underline{D}_{C} V(\varphi(t),-\dot{\varphi}(t)) \\
& =\bar{D}_{C} V(\varphi(t), \dot{\varphi}(t))=\max \left\{p \cdot \dot{\varphi}(t), p \in \partial_{C} V(\varphi(t))\right\} .
\end{aligned}
$$

This means that, for almost all $t$, the set $\left\{p \cdot \dot{\varphi}(t), p \in \partial_{C} V(\varphi(t))\right\}$ reduces to the singleton $\{(d / d t) V(\varphi(t))\}$, and then $\partial_{C} V(\varphi(t))$ is a subset of an affine subspace orthogonal to $\dot{\varphi}(t)$.

Note that, in order to prove Proposition A.3, we do not really need semiconcavity, but Lipschitz continuity and property (ii) of Proposition A.1 would be sufficient.

The following extension of second Lyapunov theorem to differential inclusions and nonpathological functions holds (see [5] for the case of $C$-regular functions, the case of nonpathological functions requires minor modifications).

Proposition A.4. Assume that $V: \mathbb{R}^{n} \rightarrow \mathbb{R}$ is positive definite, locally Lipschitz continuous, nonpathological, and radially unbounded. Assume further that

$$
\forall x \in \mathbb{R}^{n} \backslash\{0\} \quad \dot{\bar{V}}^{(A .18)}(x) \subseteq\{a \in \mathbb{R}: a<0\} .
$$

Then,

(i) (Lyapunov stability) for all $\epsilon>0$, there exists $\delta>0$ such that, for each solution $\varphi(\cdot)$ of (A.18), $|\varphi(0)|<\delta$ implies $|\varphi(t)|<\epsilon$ for all $t \geq 0$;

(ii) (attractivity) for each solution $\varphi(\cdot)$ of (A.18), $\lim _{t \rightarrow+\infty} \varphi(t)=0$.

We conclude this appendix by recalling the definition of Filippov solution used in this paper. We consider an ordinary differential equation

$$
\dot{x}=f(x), \quad x \in \mathbb{R}^{n},
$$


where $f(x)$ is locally bounded and measurable, but in general not continuous. We construct the associated differential inclusion

$$
\dot{x} \in F(x)=\bigcap_{\delta>0} \bigcap_{\mu(N)=0} \overline{\operatorname{co}}\{f(\mathscr{B}(x, \delta) \backslash N)\}
$$

(here $\mu$ is the Lebesgue measure of $\mathbb{R}^{n}, \overline{c o}$ denotes the closure of the convex hull, and $\mathscr{B}(x, r)$ is the ball of radius $r$ centered at $x)$. Finally, let $I$ be any interval. A function $\varphi(t): I \rightarrow \mathbb{R}^{n}$ is a Filippov solution of (A.24) if it is a solution in the ordinary sense of (A.25), that is, $\varphi(t)$ is absolutely continuous and satisfies $\dot{\varphi}(t) \in$ $F(\varphi(t))$ a.e. $t \in I$.

\section{B. Lemmas}

This appendix contains a number of lemmas used in the proofs of the results of this paper. For a (real or vector-valued) function $\psi(t)$, the right derivative is denoted by $\left(d^{+} / d t\right) \psi(t)$ or, when convenient, simply by $\dot{\psi}^{+}(t)$.

Lemma B.1. Let $I \subseteq \mathbb{R}$, let $\varphi: I \rightarrow \mathbb{R}^{n}$ be an absolutely continuous function, and let $V: \mathbb{R}^{n} \rightarrow \mathbb{R}$ be a locally Lipschitz continuous function. If $\bar{t} \in I$ is such that there exist both $\left(d^{+} / d t\right) V(\varphi(\bar{t}))$ and $\dot{\varphi}^{+}(\bar{t})$, then

$$
\frac{d^{+}}{d t} V(\varphi(\bar{t}))=\lim _{h \rightarrow 0^{+}} \frac{V\left(\varphi(\bar{t})+h \dot{\varphi}^{+}(\bar{t})\right)-V(\varphi(\bar{t}))}{h} .
$$

Moreover, there exists $p_{0} \in \partial_{C} V(\varphi(\bar{t}))$ such that $\left(d^{+} / d t\right) V(\varphi(\bar{t}))=p_{0} \cdot \dot{\varphi}^{+}(\bar{t})$.

Proof. The first statement is a consequence of the Lipschitz continuity of $V$ (see also [5]). As far as the second statement is concerned, we first remark that

$$
\begin{aligned}
\underline{D}_{C} V\left(\varphi(\bar{t}), \dot{\varphi}^{+}(\bar{t})\right) & \leq \underline{D}^{+} V\left(\varphi(\bar{t}), \dot{\varphi}^{+}(\bar{t})\right)=\frac{d^{+}}{d t} V(\varphi(\bar{t})) \\
& =\bar{D}^{+} V\left(\varphi(\bar{t}), \dot{\varphi}^{+}(\bar{t})\right) \leq \bar{D}_{C} V\left(\varphi(t), \dot{\varphi}^{+}(t)\right) .
\end{aligned}
$$

Then we use the fact that $\partial_{C} V(x)$ is compact and convex at each point so that the set $\left\{p \cdot \dot{\varphi}^{+}(\bar{t}), p \in \partial_{C} V(\varphi(\bar{t}))\right\}$ is a bounded and closed interval.

The following lemmas are essentially based on the dynamic programming principle. The outline of the proofs is standard, but some modifications are needed in order to face the lack of differentiability of the value function.

Lemma B.2. Let the optimal regulation problem be solvable and let $V(x)$ be its value function. Then, for each $x \in \mathbb{R}^{n}$, for each optimal solution $\varphi_{x}^{*}(\cdot)$, and for each $\bar{t} \geq 0$, the derivative $\left(d^{+} / d t\right) V\left(\varphi_{x}^{*}(\bar{t})\right)$ exists. In addition,

$$
\frac{d^{+}}{d t} V\left(\varphi_{x}^{*}(\bar{t})\right)=-\frac{1}{2} h\left(\varphi_{x}^{*}(\bar{t})\right)-\frac{\left|u_{x}^{*}(\bar{t})\right|^{2}}{2 \gamma} .
$$


1188 Nonsmooth optimal regulation and discontinuous stabilization

Proof. Let $\bar{t} \geq 0$ and let $\eta=\varphi_{x}^{*}(\bar{t})$. The solution $\varphi_{x}^{*}(t)$ for $t \geq \bar{t}$ provides an optimal trajectory issuing from $\eta$. Hence, it is sufficient to prove (B.3) for $\bar{t}=0$. Let $T>0$. Due to the definition of the value function and the dynamic programming principle, we have that

$$
\frac{V\left(\varphi_{x}^{*}(T)\right)-V(x)}{T}=-\frac{1}{2} \frac{1}{T} \int_{0}^{T}\left(h\left(\varphi_{x}^{*}(t)\right)+\frac{\left|u_{x}^{*}(t)\right|^{2}}{\gamma}\right) d t .
$$

By the continuity of $h$ and $\varphi_{x}^{*}$, and right continuity of $u_{x}^{*}(\cdot)$, there exists

$$
\lim _{T \rightarrow 0^{+}}-\frac{1}{2} \frac{1}{T} \int_{0}^{T}\left(h\left(\varphi_{x}^{*}(t)\right)-\frac{\left|u_{x}^{*}(t)\right|^{2}}{\gamma}\right) d t=-\frac{1}{2} h(x)-\frac{\left|u_{x}^{*}(0)\right|^{2}}{2 \gamma} .
$$

Then there exists also the limit on the left-hand side, that is,

$$
\lim _{T \rightarrow 0^{+}} \frac{V\left(\varphi_{x}^{*}(T)\right)-V(x)}{T}=-\frac{1}{2} h(x)-\frac{\left|u_{x}^{*}(0)\right|^{2}}{2 \gamma} .
$$

Lemma B.3. Let the value function $V(x)$ of the optimal regulation problem be locally Lipschitz continuous. Then

$$
\forall x \in \mathbb{R}^{n}, \forall u \in \mathbb{R}^{m}, \forall p \in \partial_{C} V(x), \quad p \cdot(f(x)+G(x) u) \geq-\frac{1}{2} h(x)-\frac{|u|^{2}}{2 \gamma} .
$$

Proof. We fix a control value $u_{0}$, and an instant $T>0$. Let $y$ be an arbitrary point in a neighborhood of $x$ and let $\eta=\varphi\left(T ; y, u_{0}\right)$. By the dynamic programming principle, we have that

$$
V(y) \leq \frac{1}{2} \int_{0}^{T}\left(h\left(\varphi\left(t ; y, u_{0}\right)\right)+\frac{\left|u_{0}\right|^{2}}{\gamma}\right) d t+V(\eta)
$$

and then

$$
\liminf _{T \rightarrow 0^{+}, y \rightarrow x}\left(-\frac{1}{2} \frac{1}{T} \int_{0}^{T}\left(h\left(\varphi\left(t ; y, u_{0}\right)\right)+\frac{\left|u_{0}\right|^{2}}{\gamma}\right) d t\right) \leq \liminf _{T \rightarrow 0^{+}, y \rightarrow x} \frac{V(\eta)-V(y)}{T} .
$$

We consider the two sides of the inequality separately.

Left-hand side. There exists $\theta \in[0, T]$ such that

$$
-\frac{1}{2} \frac{1}{T} \int_{0}^{T}\left(h\left(\varphi\left(t ; y, u_{0}\right)\right)+\frac{\left|u_{0}\right|^{2}}{\gamma}\right) d t=-\frac{1}{2} h\left(\varphi\left(\theta ; y, u_{0}\right)\right)-\frac{\left|u_{0}\right|^{2}}{2 \gamma} .
$$


By continuous dependence of solutions of Cauchy problem on initial data, we have that $\liminf _{T \rightarrow 0^{+}, y \rightarrow x} \varphi\left(\theta ; y, u_{0}\right)=x$, and then

$$
\liminf _{T \rightarrow 0^{+}, y \rightarrow x}\left(-\frac{1}{2} \frac{1}{T} \int_{0}^{T}\left(h\left(\varphi\left(t ; y, u_{0}\right)\right)+\frac{\left|u_{0}\right|^{2}}{\gamma}\right) d t\right)=-\frac{1}{2} h(x)-\frac{\left|u_{0}\right|^{2}}{2 \gamma} .
$$

Right-hand side. We consider the quotient

$$
\begin{aligned}
\frac{V\left(\varphi\left(T ; y, u_{0}\right)\right)-V(y)}{T}= & \frac{V\left(\varphi\left(T ; y, u_{0}\right)\right)-V\left(y+\left(f(x)+G(x) u_{0}\right) T\right)}{T} \\
& +\frac{V\left(y+\left(f(x)+G(x) u_{0}\right) T\right)-V(y)}{T} .
\end{aligned}
$$

By Lipschitz continuity of $V$, there exists $L>0$ such that

$$
\begin{aligned}
& \left|\frac{V\left(\varphi\left(T ; y, u_{0}\right)\right)-V\left(y+\left(f(x)+G(x) u_{0}\right) T\right)}{T}\right| \\
& \leq \frac{L}{T}\left|\varphi\left(T ; y, u_{0}\right)-y-\left(f(x)+G(x) u_{0}\right) T\right| \\
& \quad=\frac{L}{T} \mid x+\frac{\partial \varphi}{\partial t}\left(0 ; x, u_{0}\right) T+\frac{\partial \varphi}{\partial y}\left(0 ; x, u_{0}\right) \cdot(y-x)+o(T,|y-x|) \\
& \quad \quad-y-\left(f(x)+G(x) u_{0}\right) T \mid \\
& \quad \leq \frac{L}{T} o(T,|y-x|) .
\end{aligned}
$$

Then there exists

$$
\lim _{T \rightarrow 0^{+}, y \rightarrow x} \frac{V\left(\varphi\left(T ; y, u_{0}\right)\right)-V\left(y+\left(f(x)+G(x) u_{0}\right) T\right)}{T}=0 .
$$

Coming back to (B.12), we therefore have

$$
\begin{aligned}
\liminf _{T \rightarrow 0^{+}, y \rightarrow x} & \frac{V\left(\varphi\left(T ; y, u_{0}\right)\right)-V(y)}{T} \\
\quad= & \liminf _{T \rightarrow 0^{+}, y \rightarrow x} \frac{V\left(y+\left(f(x)+G(x) u_{0}\right) T\right)-V(y)}{T} \\
\quad= & \underline{D}_{C} V\left(x, f(x)+G(x) u_{0}\right) \\
& =\min \left\{p \cdot\left(f(x)+G(x) u_{0}\right), p \in \partial_{C} V(x)\right\} .
\end{aligned}
$$

By comparing the two sides of inequality (B.9), the conclusion of the lemma follows. 
LеммA B.4. If the optimal regulation problem is solvable and its value function is locally Lipschitz continuous, then

$$
\forall x, \forall p \in \underline{\partial} V(x), \quad p \cdot\left(f(x)+G(x) u_{x}^{*}(0)\right)=-\frac{1}{2} h(x)-\frac{\left|u_{x}^{*}(0)\right|^{2}}{2 \gamma} .
$$

Proof. We consider $\left(d^{+} / d t\right) V\left(\varphi_{x}^{*}(t)\right)$. On the one hand, by Lemma B.2, we have that

$$
\frac{d^{+}}{d t} V\left(\varphi_{x}^{*}(0)\right)=-\frac{1}{2} h(x)-\frac{\left|u_{x}^{*}(0)\right|^{2}}{2 \gamma}
$$

On the other hand, from the characterization of the subdifferential by means of the contingent derivatives (see Appendix A) and Lemma B.1, it follows that, for all $p \in \underline{\partial} V(x)$,

$$
\begin{aligned}
p \cdot( & \left.f(x)+G(x) u_{x}^{*}(0)\right) \\
& \leq \underline{D}_{K}^{+} V\left(x, f(x)+G(x) u_{x}^{*}(0)\right)=\underline{D}^{+} V\left(x, f(x)+G(x) u_{x}^{*}(0)\right) \\
& =\liminf _{t \rightarrow 0^{+}} \frac{V\left(x+t\left(f(x)+G(x) u_{x}^{*}(0)\right)\right)-V(x)}{t}=\frac{d^{+}}{d t} V\left(\varphi_{x}^{*}(0)\right) .
\end{aligned}
$$

Finally, we get that, for all $x$ and for all $p \in \underline{\partial} V(x)$,

$$
p \cdot\left(f(x)+G(x) u_{x}^{*}(0)\right) \leq-\frac{1}{2} h(x)-\frac{\left|u_{x}^{*}(0)\right|^{2}}{2 \gamma} .
$$

Since $\underline{\partial} V(x) \subseteq \partial_{C} V(x)$, the opposite inequality is provided by Lemma B.3.

In the next lemma, we denote by $\mathcal{K}_{0}$ the class of functions $a:[0,+\infty) \rightarrow$ $[0,+\infty)$ such that $a(\cdot)$ is continuous, strictly increasing, and $a(0)=0$.

Lemma B.5. Let $x_{0}$ be fixed. Let $a \in \mathcal{K}_{0}$ be such that $h(x) \geq a(|x|)$ for each $x \in \mathbb{R}^{n}$ (such a function exists if $h$ is continuous and positive definite). Assume also that $J\left(x_{0}, u(\cdot)\right)<\infty$ for some measurable, locally bounded input $u(t)$. Then,

$$
\lim _{t \rightarrow+\infty} \varphi\left(t ; x_{0}, u(\cdot)\right)=0
$$

Proof. Since $u(t)$ and $x_{0}$ are fixed, we will write simply $\varphi(t)$ instead of $\varphi\left(t ; x_{0}\right.$, $u(\cdot))$. From the assumption, it follows that both the integrals

$$
\int_{0}^{+\infty} h(\varphi(t)) d t, \quad \int_{0}^{+\infty}|u(t)|^{2} d t
$$

converge. It follows in particular that $u(t)$ is square integrable on $[0,+\infty)$ and on every subinterval of $[0,+\infty)$. It also follows that $\liminf _{t \rightarrow+\infty} h(\varphi(t))=0$, and 
since $h(x)$ is continuous, positive definite, and bounded from below by a class$\mathcal{K}_{0}$ function, this in turn implies that

$$
\liminf _{t \rightarrow+\infty}|\varphi(t)|=0
$$

Assume, by contradiction, that $\lim \sup _{t \rightarrow+\infty}|\varphi(t)|>0$, and let

$$
l=\min \left\{1, \limsup _{t \rightarrow+\infty}|\varphi(t)|\right\} \text {. }
$$

Let $L$ be a Lipschitz constant for $f(x)$, valid on the sphere $|x| \leq l$. Moreover, let $b>0$ be a bound for the norm of the matrix $G(x)$ for $|x| \leq l$. By the definition of $l$, there exists a strictly increasing, divergent sequence $\left\{t_{j}\right\}$ such that for each $j$,

$$
\left|\varphi\left(t_{j}\right)\right|>\frac{3 l}{4}
$$

Without loss of generality, we can assume that $t_{j+1}-t_{j}>1 /(4 L)$ for each $j \in$ $\mathbb{N}$. The existence of some $\tau>0$ and $k \in \mathbb{N}$, such that, for each $j>k$ and each $t \in\left[t_{j}-\tau, t_{j}\right]$

$$
\|\varphi(t)\| \geq \frac{l}{4}
$$

is excluded since in this case the first integral in (B.21) would be divergent (here again, we use the fact that $h$ is bounded from below by $a(\cdot))$. Hence, for $\tau=$ $1 /(4 L)$ and each $k \in \mathbb{N}$, we can find an index $j_{k}>k$ and an instant $s_{k}$ such that

$$
t_{j_{k}}-\tau \leq s_{k} \leq t_{j_{k}}, \quad\left|\varphi\left(s_{k}\right)\right|<\frac{l}{4} .
$$

Since the solution $\varphi(t)$ is continuous, for each $k$, there exist two instants $\sigma_{k}$, $\theta_{k}$ such that

$$
\begin{gathered}
s_{k}<\sigma_{k}<\theta_{k}<t_{j_{k}}, \quad\left|\varphi\left(\sigma_{k}\right)\right|=\frac{l}{4}, \quad\left|\varphi\left(\theta_{k}\right)\right|=\frac{3 l}{4}, \\
\frac{l}{4}<|\varphi(t)|<\frac{3 l}{4}, \quad \forall t \in\left(\sigma_{k}, \theta_{k}\right) .
\end{gathered}
$$

We have, for each $k$,

$$
\left|\varphi\left(\theta_{k}\right)-\varphi\left(\sigma_{k}\right)\right| \geq\left|\varphi\left(\theta_{k}\right)\right|-\left|\varphi\left(\sigma_{k}\right)\right|=\frac{l}{2} .
$$

On the other hand,

$$
\left|\varphi\left(\theta_{k}\right)-\varphi\left(\sigma_{k}\right)\right| \leq \int_{\sigma_{k}}^{\theta_{k}}|f(\varphi(t))| d t+\int_{\sigma_{k}}^{\theta_{k}}|G(\varphi(t))| \cdot|u(t)| d t .
$$


1192 Nonsmooth optimal regulation and discontinuous stabilization

By construction, for $t \in\left[\sigma_{k}, \theta_{k}\right]$, we have $|\varphi(t)| \leq l$. Hence, on the interval $\left[\sigma_{k}, \theta_{k}\right]$, the following inequalities hold:

$$
|f(\varphi(t))| \leq L|\varphi(t)|, \quad|G(x)| \leq b .
$$

This yields

$$
\left|\varphi\left(\theta_{k}\right)-\varphi\left(\sigma_{k}\right)\right| \leq l L \tau+b \int_{t_{j_{k}}-\tau}^{t_{j_{k}}}|u(t)| d t .
$$

Now, taking into account (B.28) and (B.31), and recalling that $\tau=1 /(4 L)$, we infer that

$$
\frac{l}{2} \leq \frac{l}{4}+b \int_{t_{j_{k}}-\tau}^{t_{j_{k}}}|u(t)| d t
$$

that is,

$$
\int_{t_{j_{k}}-\tau}^{t_{j_{k}}}|u(t)| d t \geq \frac{l}{4 b} .
$$

Using Hölder inequality and the fact that $u(t)$ is square-integrable on $\left[t_{j_{k}}-\right.$ $\left.\tau, t_{j_{k}}\right]$, we also have

$$
C\left(\int_{t_{j_{k}}-\tau}^{t_{j_{k}}}|u(t)|^{2} d t\right)^{1 / 2} \geq \int_{t_{j_{k}}-\tau}^{t_{j_{k}}}\|u(t)\| d t
$$

where $C$ is a positive constant independent of $u(\cdot)$. This yields

$$
\int_{t_{j_{k}}-\tau}^{t_{j_{k}}}|u(t)|^{2} d t \geq \frac{l^{2}}{16 b^{2} C^{2}}>0
$$

But this is impossible since $u(t)$ is square-integrable on $[0,+\infty)$ by virtue of (B.21). Thus, we conclude that

$$
\liminf _{t \rightarrow+\infty}|\varphi(t)|=\limsup _{t \rightarrow+\infty}|\varphi(t)|=0,
$$

which implies that

$$
\lim _{t \rightarrow+\infty}|\varphi(t)|=0
$$

as required.

Lemma B.5 is reminiscent of the so-called Barbalat's lemma [27, page 491]. However, it does not reduce to Barbalat's lemma since we have to take into account the input variable and we cannot use uniform continuity of solutions. 
Lemma B.6. Let $V(x): \mathbb{R}^{n} \rightarrow \mathbb{R}$ be locally Lipschitz continuous and nonpathological. Let $x \in \mathbb{R}^{n}$ and let $u(\cdot)$ be any admissible input. For simplicity, write $\varphi(t)=$ $\varphi(t ; x, u(\cdot))$. Finally, let $p(t)$ be any measurable function such that $p(t) \in \partial_{C} V(\varphi(t))$ a.e. Then,

$$
\int_{t_{1}}^{t_{2}} p(t) \cdot \dot{\varphi}(t) d t=V\left(\varphi\left(t_{2}\right)\right)-V\left(\varphi\left(t_{1}\right)\right)
$$

Proof. Under our assumptions, for a.e. $t \in \mathbb{R}$, there exists $p_{0} \in \partial_{C} V(\varphi(t))$ such that the right derivative $\left(d^{+} / d t\right) V(\varphi(t))$ exists and it is equal to $p_{0} \cdot\left(d^{+} / d t\right) \varphi(t)$ (see Lemma B.1). The conclusion follows by virtue of the definition of nonpathological function.

\section{References}

[1] F. Ancona and A. Bressan, Patchy vector fields and asymptotic stabilization, ESAIM Control Optim. Calc. Var. 4 (1999), 445-471.

[2] B. D. O. Anderson and J. B. Moore, Linear Optimal Control, Prentice-Hall, New Jersey, 1971.

[3] Z. Artstein, Stabilization with relaxed controls, Nonlinear Anal. 7 (1983), no. 11, $1163-1173$.

[4] J.-P. Aubin and H. Frankowska, Set-Valued Analysis, Systems \& Control: Foundations \& Applications, vol. 2, Birkhäuser Boston, Massachusetts, 1990.

[5] A. Bacciotti and F. Ceragioli, Stability and stabilization of discontinuous systems and nonsmooth Lyapunov functions, ESAIM Control Optim. Calc. Var. 4 (1999), 361376.

[6] _ Optimal regulation and discontinuous stabilization, Proceedings NOLCOS '01, Elsevier Sci., St. Petersburg, 2001, pp. 36-41.

[7] M. Bardi and I. Capuzzo-Dolcetta, Optimal Control and Viscosity Solutions of Hamilton-Jacobi-Bellman Equations, Systems \& Control: Foundations \& Applications, Birkhäuser Boston, Massachusetts, 1997.

[8] D. S. Bernstein, Nonquadratic cost and nonlinear feedback control, Internat. J. Robust Nonlinear Control 3 (1993), no. 3, 211-229.

[9] R. W. Brockett, Asymptotic stability and feedback stabilization, Differential Geometric Control Theory (Houghton, Mich, 1982) (R. W. Brockett, R. S. Millman, and H. J. Sussmann, eds.), Progr. Math., vol. 27, Birkhäuser Boston, Massachusetts, 1983, pp. 181-191.

[10] P. Cannarsa and G. Da Prato, Nonlinear optimal control with infinite horizon for distributed parameter systems and stationary Hamilton-Jacobi equations, SIAM J. Control Optim. 27 (1989), no. 4, 861-875.

[11] P. Cannarsa and C. Sinestrari, Convexity properties of the minimum time function, Calc. Var. Partial Differential Equations 3 (1995), no. 3, 273-298.

[12] P. Cannarsa and H. M. Soner, On the singularities of the viscosity solutions to Hamilton-Jacobi-Bellman equations, Indiana Univ. Math. J. 36 (1987), no. 3, 501524.

[13] F. H. Clarke, Optimization and Nonsmooth Analysis, Canadian Mathematical Society Series of Monographs and Advanced Texts, John Wiley \& Sons, New York, 1983.

[14] F. H. Clarke, Yu. S. Ledyaev, L. Rifford, and R. J. Stern, Feedback stabilization and Lyapunov functions, SIAM J. Control Optim. 39 (2000), no. 1, 25-48. 
[15] F. H. Clarke, Yu. S. Ledyaev, E. D. Sontag, and A. I. Subbotin, Asymptotic controllability implies feedback stabilization, IEEE Trans. Automat. Control 42 (1997), no. 10, 1394-1407.

[16] F. H. Clarke, Yu. S. Ledyaev, R. J. Stern, and P. R. Wolenski, Nonsmooth Analysis and Control Theory, Graduate Texts in Mathematics, vol. 178, Springer-Verlag, New York, 1998.

[17] F. H. Clarke, L. Rifford, and R. J. Stern, Feedback in state constrained optimal control, ESAIM Control Optim. Calc. Var. 7 (2002), 97-133.

[18] R. Conti, Linear Differential Equations and Control, Institutiones Mathematicae, vol. 1, Academic Press, London, 1976.

[19] F. Da Lio, On the Bellman equation for infinite horizon problems with unbounded cost functional, Appl. Math. Optim. 41 (2000), no. 2, 171-197.

[20] A. F. Filippov, Differential Equations with Discontinuous Righthand Sides, Mathematics and Its Applications (Soviet Series), vol. 18, Kluwer Academic, Dordrecht, 1988.

[21] H. Frankowska, Optimal trajectories associated with a solution of the contingent Hamilton-Jacobi equation, Appl. Math. Optim. 19 (1989), no. 3, 291-311.

[22] S. T. Glad, On the gain margin of nonlinear and optimal regulators, IEEE Trans. Automat. Control 29 (1984), no. 7, 615-620.

[23] H. Hermes, Stabilization via optimization, New Trends in Systems Theory (Genoa, 1990) (G. Conte, A.-M. Perdon, and B. Wyman, eds.), Progr. Systems Control Theory, vol. 7, Birkhäuser Boston, Massachusetts, 1991, pp. 379-385.

[24] Resonance, stabilizing feedback controls, and regularity of viscosity solutions of Hamilton-Jacobi-Bellman equations, Math. Control Signals Systems 9 (1996), no. 1, 59-72.

[25] D. H. Jacobson, Extensions of Linear-Quadratic Control, Optimization and Matrix Theory, Mathematics in Science and Engineering, vol. 133, Academic Press, London, 1977.

[26] M. Janković, R. Sepulchre, and P. V. Kokotovic, CLF based designs with robustness to dynamic input uncertainties, Systems Control Lett. 37 (1999), no. 1, 45-54.

[27] M. Krstic, I. Kanellakopoulos, and P. V. Kokotovic, Nonlinear and Adaptive Control Design, John Wiley \& Sons, New York, 1995.

[28] E. B. Lee and L. Markus, Foundations of Optimal Control Theory, John Wiley \& Sons, New York, 1967.

[29] P. J. Moylan and B. D. O. Anderson, Nonlinear regulator theory and an inverse optimal control problem, IEEE Trans. Automat. Control 18 (1973), no. 5, 460-465.

[30] B. E. Paden and S. S. Sastry, A calculus for computing Filippov's differential inclusion with application to the variable structure control of robot manipulators, IEEE Trans. Circuits and Systems 34 (1987), no. 1, 73-82.

[31] L. Rifford, Existence of Lipschitz and semiconcave control-Lyapunov functions, SIAM J. Control Optim. 39 (2000), no. 4, 1043-1064.

[32] On the existence of nonsmooth control-Lyapunov functions in the sense of generalized gradients, ESAIM Control Optim. Calc. Var. 6 (2001), 593-611.

[33] R. Sepulchre, M. Janković, and P. V. Kokotovic, Constructive Nonlinear Control, Communications and Control Engineering Series, Springer-Verlag, Berlin, 1997.

[34] D. Shevitz and B. E. Paden, Lyapunov stability theory of nonsmooth systems, IEEE Trans. Automat. Control 39 (1994), no. 9, 1910-1914.

[35] E. D. Sontag, Mathematical Control Theory, 2nd ed., Texts in Applied Mathematics, vol. 6, Springer-Verlag, New York, 1998. 
[36] - Stability and stabilization: discontinuities and the effect of disturbances, Nonlinear Analysis, Differential Equations and Control (Montreal, Quebec, 1998) (F. H. Clarke, R. J. Stern, and G. Sabidussi, eds.), NATO Sci. Ser. C Math. Phys. Sci., vol. 528, Kluwer Academic Publishers, Dordrecht, 1999, pp. 551-598.

[37] J. N. Tsitsiklis and M. Athans, Guaranteed robustness properties of multivariable nonlinear stochastic optimal regulators, IEEE Trans. Automat. Control 29 (1984), no. 8, 690-696.

[38] M. Valadier, Entraînement unilatéral, lignes de descente, fonctions lipschitziennes non pathologiques [Unilateral driving, descent curves, nonpathological Lipschitzian functions], C. R. Acad. Sci. Paris Sér. I Math. 308 (1989), no. 8, 241-244 (French).

A. Bacciotti: Dipartimento di Matematica, Politecnico di Torino, Corso Duca degli Abruzzi 24, 10129 Torino, Italy

E-mail address: bacciotti@polito.it

F. Ceragioli: Dipartimento di Matematica, Politecnico di Torino, Corso Duca degli Abruzzi 24, 10129 Torino, Italy

E-mail address: ceragiol@calvino.polito.it 


\section{Differential Equations \& Nonlinear Mechanics}

\section{An Open Access Journal}

\section{Editor-in-Chief}

K. Vajravelu

USA

Associate Editors

N. Bellomo

Italy

J. L. Bona

USA

J. R. Cannon

USA

S.-N. Chow

USA

B. S. Dandapat

India

E. DiBenedetto

USA

R. Finn

USA

R. L. Fosdick

USA

J. Frehse

Germany

A. Friedman

USA

R. Grimshaw

UK

J. Malek

Czech Republic

J. T. Oden

USA

R. Quintanilla

Spain

K. R. Rajagopal

USA

G. Saccomandi

Italy

Y. Shibata

Japan

Ivar Stakgold

USA

Swaroop Darbha

USA

A. Tani

Japan

S. Turek

Germany

A. Wineman

USA
Website: http://www.hindawi.com/journals/denm/

Aims and Scope

Differential equations play a central role in describing natural phenomena as well as the complex processes that arise from science and technology. Differential Equations \& Nonlinear Mechanics (DENM) will provide a forum for the modeling and analysis of nonlinear phenomena. One of the principal aims of the journal is to promote cross-fertilization between the various subdisciplines of the sciences: physics, chemistry, and biology, as well as various branches of engineering and the medical sciences.

Special efforts will be made to process the papers in a speedy and fair fashion to simultaneously ensure quality and timely publication.

DENM will publish original research papers that are devoted to modeling, analysis, and computational techniques. In addition to original full-length papers, DENM will also publish authoritative and informative review articles devoted to various aspects of ordinary and partial differential equations and their applications to sciences, engineering, and medicine.

\section{Open Access Support}

The Open Access movement is a relatively recent development in academic publishing. It proposes a new business model for academic publishing that enables immediate, worldwide, barrier-free, open access to the full text of research articles for the best interests of the scientific community. All interested readers can read, download, and/or print any Open Access articles without requiring a subscription to the journal in which these articles are published.

In this Open Access model, the publication cost should be covered by the author's institution or research funds. These Open Access charges replace subscription charges and allow the publishers to give the published material away for free to all interested online visitors.

\section{Instructions for Authors}

Original articles are invited and should be submitted through the DENM manuscript tracking system at http://www.mstracking.com/ denm/. Only pdf files are accepted. If, for some reason, submission through the manuscript tracking system is not possible, you can contact denm.support@hindawi.com.

Hindawi Publishing Corporation

410 Park Avenue, 15th Floor, \#287 pmb, New York, NY 10022, USA
HINDAWI 


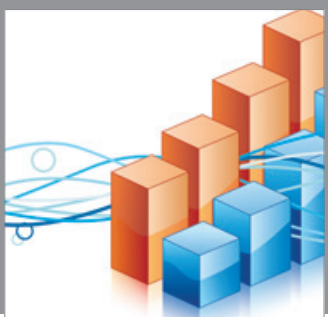

Advances in

Operations Research

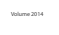

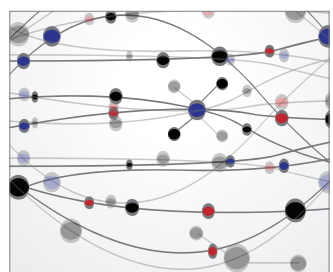

\section{The Scientific} World Journal
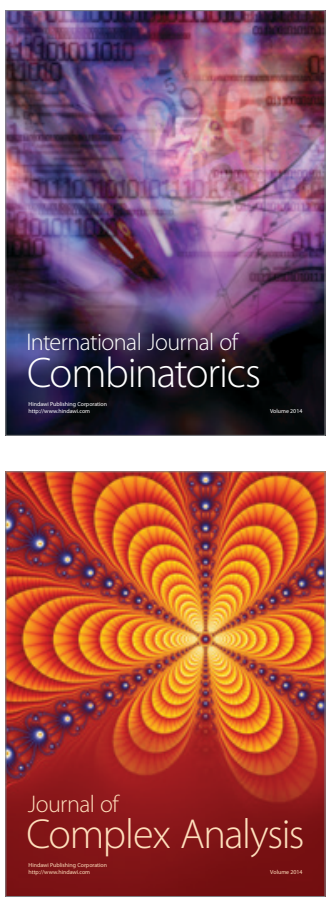

International Journal of

Mathematics and

Mathematical

Sciences
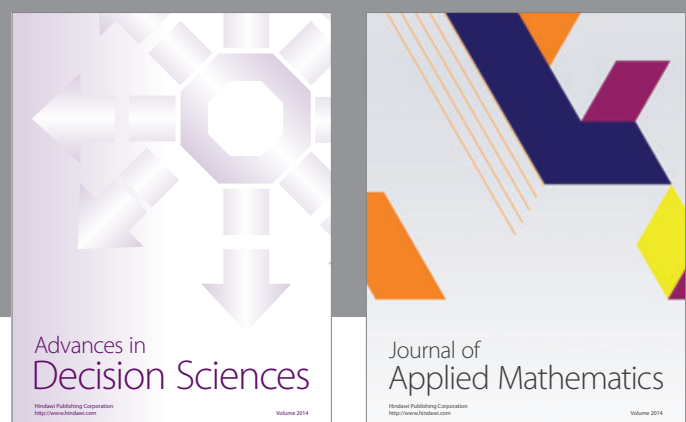

Journal of

Applied Mathematics
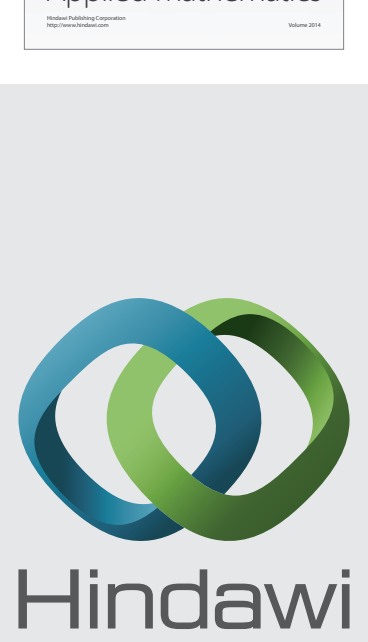

Submit your manuscripts at http://www.hindawi.com
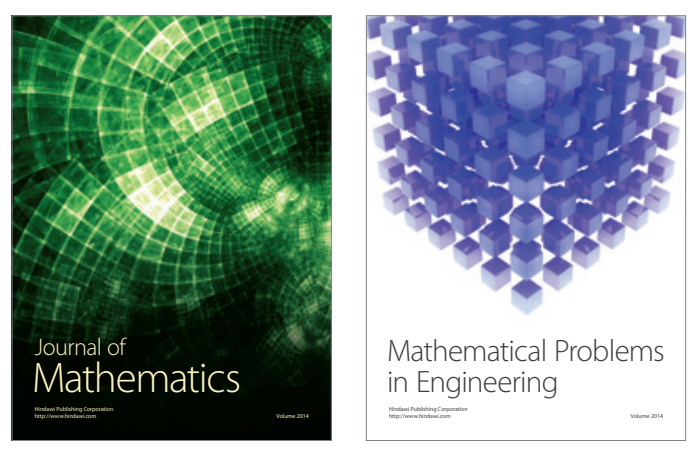

Mathematical Problems in Engineering
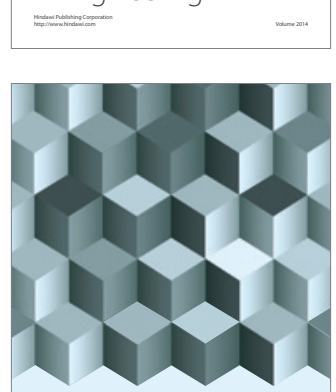

Journal of

Function Spaces
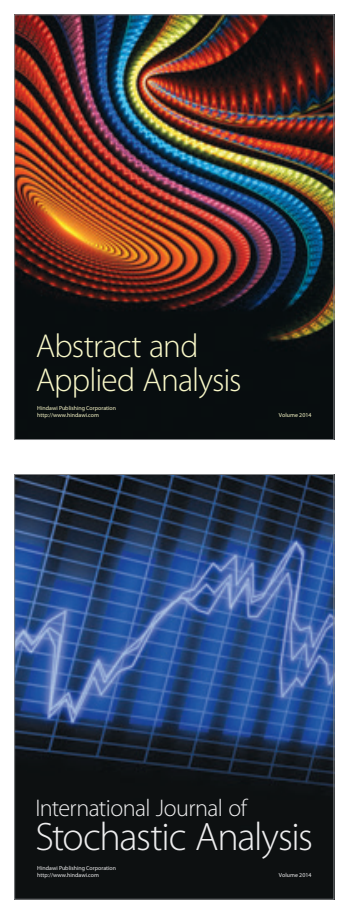

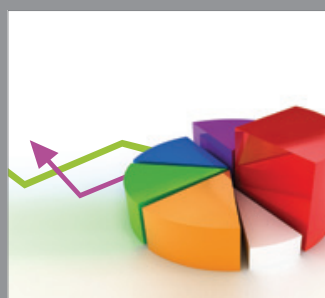

ournal of

Probability and Statistics

Promensencen
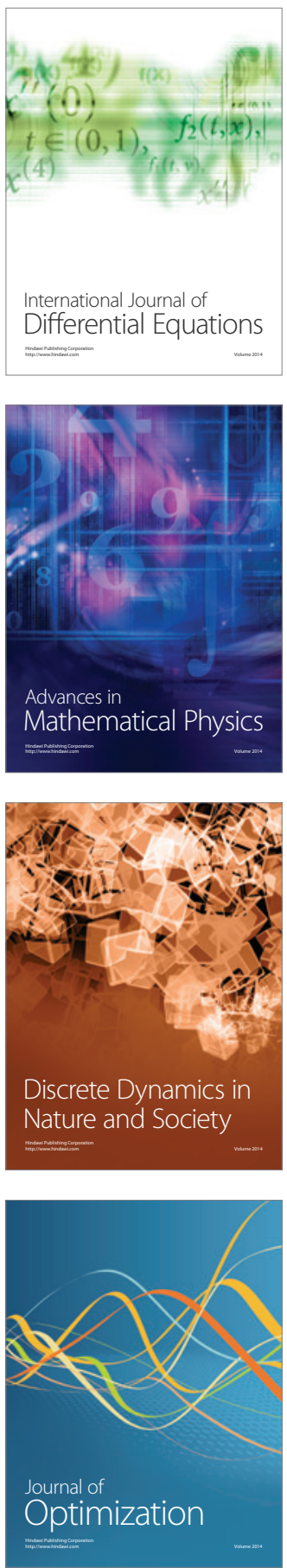